\title{
On the spectrum of a stretched spiral vortex
}

\author{
D. I. Pullin \\ Graduate Aeronautical Laboratories 105-50, C'alifornia Institute of Technology, Pasadena, California 91125
}

James D. Buntine and P. G. Saffman

Applied Mathematics 217-50, California Institute of Technology, Pasadena, California 91125

(Received 2 December 1993; accepted 9 May 1994)

Corrections are found to the $k^{-5 / 3}$ spectrum of Lundgren [Phys. Fluids 25, 2193 (1982)] for a stretched spiral vortex model ( $a$ is the stretching strain rate and $k$ the scalar wave number) of turbulent fine scales. These take the form of additional terms arising from the early time evolution, when the stretching of vortex lines is small. For the special case when the spiral takes the form of a rolled-up shear layer, it is shown that the composite spectrum is divergent, thus requiring the introduction of a finite early cutoff time $\tau_{1}$ in the time integral for the nonaxisymmetric contribution. The identity $\nu \overline{\omega^{2}}=2 \nu \int_{0}^{\infty} k^{2} E(k) d k$ which gives the dissipation is then satisfied self-consistently. Direct numerical calculation of the energy spectrum from the approximate vorticity field for a special choice of spiral structure nevertheless indicates that the one-term $k^{-5 / 3}$-spectrum result is asymptotically valid in the inertial range provided $a \tau_{1}$ is $O(1)$ but that the numerically calculated dissipation spectrum appears to lie somewhere between an $\exp \left(-B_{1} k^{2}\right)$ and an $\exp \left(-B_{2} k\right)$ form. It is also shown that the stretched, rolled-up shear-layer model predicts asymptotic shell-summed spectra of the energy dissipation and of the square of the vorticity, each asymptotically constant, with no power-law dependence, for $k$ smaller than the Kolmogorov wave number. The corresponding one-dimensional spectra each show $-\log \left(k_{1}\right)$ behavior for small $k_{1}$. The extension of the model given by Pullin and Saffman [Phys. Fluids A 5, 126 (1993)] is reformulated by the introduction of a long-time cutoff in the vortex lifetime and an additional requirement that the vortex structures be approximately space filling. This gives a reduction in the number of model free-parameters but introduces a dependence of the calculated Kolmogorov constant and skewness on the ratio of the initial vortex radius to the equivalent Burgers-vortex radius. A scaling for this ratio in terms of the Taylor microscale Reynolds number is proposed in which the stretching strain is assumed to be provided by the large scales with spatial coherence limited to the maximum stretched length of the structures. Postdictions of the fourth-order flatness factor and of higher moments of the longitudinal velocity gradient statistics are compared with numerical simulation.

\section{INTRODUCTION}

Lundgren ${ }^{1}$ henceforth referred to as $L$, proposed a model of turbulent fine scales in which the fine structure consisted of a collection of stretched spiral vortices described by approximate solutions of the Navier-Stokes equations. Each vortex is subject to an axisymmetric external strain field which is supposed produced by the local strain-rate field of larger scales. It is assumed that these structures are created by a process extcrnal to the model, either by vortex amalgamation or instability of larger scales, or by some other mechanism such that the production of vortex length per unit time per unit volume is a quantity $N$ with dimensions length ${ }^{-2} \times$ time. $^{-1}$ The strain field, although assumed to be uniform and constant with strain-rate $a$ is to be viewed as a statistical average of more complex strain histories experienced by all members of the collection. In $L$ it is shown that an ensemble of such spiral vortices has a $k^{-5 / 3}$ range in the energy spectrum, independent of the detailed structure of the spiral.

Pullin and Saffman, ${ }^{2}$ henceforth referred to as PS, considered a particular realization of the spiral vortex which took the form of a thin shear layer in the process of roll-up. We emphasize here and elsewhere that this is a special case of a more general structure proposed by $L$. In the PS model, all vortices comprising the small scale structure have the same initial radius $R$ and circulation scale $\Gamma_{0}$ which may be interpreted as averages, over unknown statistics, of distributions of these quantities. This defines three dimensionless groupings; a production rate parameter $N R^{2} / a$, a vortex Reynolds number $\Gamma_{0} / \nu$, where $\nu$ is the kinematic viscosity, and the ratio $a R^{2} / 4 \nu$ of $R^{2}$ to the squared radius $4 \nu / a$ of an equivalent Burgers vortex. To these PS added the Kolmogorov prefactor $Z_{0}$, the skewness $S_{3}$ and a parameter $a^{2} \nu / \epsilon$, where $\epsilon$ is the average energy dissipation per unit mass. For these six quantities three equations, $\operatorname{PS}(37), \operatorname{PS}(38)$, and PS(40) were obtained. These were independent of $a R^{2} / 4 \nu$ and PS proceeded by solving for $a^{2} \nu / \epsilon, \mathbb{K}_{0}$ and $S_{3}$ with fixed values of $\Gamma_{0} / \nu=100$ and $N R^{2} / a=0.6$ chosen to give values $\mathscr{K}_{0}$ and $S_{3}$ in the range of experiment. An important constraint was the upper bound on $\Gamma_{0} / \nu$ of $O(100)$ required to ensure that when an upper cutoff time in the vortex evolution, $\tau_{2}$, was set to $\infty$, the composite spectrum was not dominated by the axisymmetric component in such a way as to obscure the $k^{-5 / 3}$ inertial range. PS calculated, directly from the assumed vorticity field, higher-order moments of the longitudinal and lateral velocity derivatives, and of one component of the vorticity. The $2 p$ th vorticity moment, reproduced presently as Eq. (71), showed a $\left(a R^{2} / 4 \nu\right)^{p-3 / 2}$ 
dependence for $p \geqslant 2$. In order to introduce a Taylor Reynolds number dependence PS proposed a closure assumption

$$
R^{2}=\lambda\left(\frac{4 \nu}{a}\right)^{1 / 2},
$$

where $\lambda$ is the Taylor microscale. This gave the result that the $2 p$ th vorticity moment and the $2 p$ th velocity derivative moments varied as $R_{\lambda}^{p / 2-3 / 4}, p \geqslant 2$, where $R_{\lambda}$ is the Taylor Reynolds number to be defined precisely in Sec. V.

The PS analysis is subject to several shortcomings. First they (Sec. VIC) found that the kinematic identity $\overline{\omega^{2}}=2 \int_{0}^{\infty} k^{2} E(k) d k$ is not satisfied, where the overbar, and double overbar notation used presently is in accordance with PS(50). Second their value of $\Gamma_{0} / \nu$ is rather smaller than found in measurements of vortex tubes by Jiménez $z^{3}$ et al. in numerical simulations of isotropic turbulence. Third, numerical simulations of the Lundgren-spiral vortex by Lundgren ${ }^{4}$ and presently indicate that the PS value of $\Gamma / \nu$ is too small to produce an inertial range as predicted by the $L$ asymptotics. Fourth, PS allowed individual vortices to evolve for infinite time, so that continuous production of vortex tube length at rate per unit volume $N$ must necessarily overfill space. Fifth, as pointed out by Kida, ${ }^{5} \mathrm{Eq}$. (1) limits any inertial range of the model to rather large $k$. Sixth, the closure given by Eq. (1) was motivated not by physical argument but by interpretations placed by PS on observations of vortex-tube dimensions made by Vincent and Meneguzzi ${ }^{6}$ from numerical simulations of $240^{3}$ isotropic turbulence. Seventh, the Gaussian decay of the energy spectrum in the dissipation range does not agree with the exponential decay found experimentally and in simulations.

In the present paper we extend the PS formulation in an attempt to address these difficulties. First it is shown in Sec. II that the $L$ analysis captures only one component of the asymptotic spectrum for the strained spiral arising for $a \tau$ large [ $\tau$ is a stretched time variable described by Eq. (6)]. For the form of the spiral shear-layer considered by PS, there exists an early period in the spiral evolution with $a \tau \leqslant 1$ which contributes a leading-order spectral term of the form $k^{-2} E_{1}\left[2 \phi k^{2} \nu / 3 a\right]$, where $\tau_{1}=\phi / a$ is a lower cutoff time in the time integral and $E_{1}[z]$ is the exponential integral [Abramowitz and Stegun, ${ }^{7}$ Eq. (5.1.1)]. Thus the composite spectrum is divergent in the sense of unbounded dissipation unless $\phi>0$. In Sec. III we calculate the shell-summed spectra of the dissipation and of the square of the vorticity for the model. Section IV describes an attempt to calculate the spectrum from numerical evaluation of the spectrum integrals for the particular PS realization of the $L$ spiral as a test of the asymptotic theory.

In Sec. V the form of the model given by PS is reformulated. We introduce an additional requirement, namely, that the vortex structures be approximately space filling, together with a new assumption that there exists a finite upper cutoff $\tau_{2}$ which limits the growth of the core and so allows an increase in the model $\Gamma_{0} / \nu$ while retaining a visible inertial range. The extended model is shown to have internal selfconsistency through agreement of the skewness calculated by two independent methods, the first of which is essentially kinematic and the second of which makes use of the (dy- namical) Kármán-Howarth equation. Further, an alternative to Eq. (1) is proposed based on the idea that the vortex structures are initially $O(R)$ in length as well as in lateral extent, and that the large-scale strain $a$ is coherent only up to length scales that correspond to the length of the stretched vortex at $\tau_{2}$. New postdictions of the fourth-order flatness factor and of some higher order moments of the velocity-gradient field are discussed.

\section{SPECTRUM OF THE SPIRAL}

\section{A. The Lundgren analysis}

In $L$ each vortex is subject to an axisymmetric external strain field, with velocity in cylindrical polar coordinates $(r, \theta, z)$ given by $\left(u_{r}, u_{\theta}, u_{z}\right)=(-a r / 2,0, a z)$, where $a$ is the constant ratc of strain. The vorticity is $\left(\omega_{r}, \omega_{\theta}, \omega_{z}\right)$ $=[0,0, \omega(r, \theta, t)]$, where $t$ is the time. There is alignment of vorticity with the positive principal rate of strain of the external field; $L$ gives a rather general asymptotic solution of the Navier-Stokes equations describing the spiral which we write as

$$
\begin{aligned}
& \omega(r, \theta, t)=e^{a t} \hat{\omega}(\rho, \theta, \tau), \\
& \hat{\omega}(\rho, \theta, \tau)=\sum_{-\infty}^{\infty} \hat{\omega}_{n}(\rho, \tau) \exp (\operatorname{in} \theta), \\
& \hat{\omega}_{n}(\rho, \tau)=f_{n}(\rho) \exp \left[-i n \Omega(\rho) \tau-\nu n^{2} \Lambda^{2}(\rho) \tau^{3} / 3\right], \\
& n \neq 0 \text {, } \\
& \hat{\omega}_{0}(\rho, \tau)=g(\rho, \tau)+f_{0}(\rho, \tau), \\
& \rho(r, t)=r e^{a t / 2}, \quad \tau(t)=\left(e^{a t}-1\right) / a,
\end{aligned}
$$

where

$$
\begin{aligned}
& \frac{1}{\rho} \frac{d}{d \rho}\left[\rho^{2} \Omega(\rho)\right]=g(\rho)+f_{0}(\rho), \\
& \Lambda=\frac{d \Omega}{d \rho} .
\end{aligned}
$$

In Eqs. (2) $-(8),(\rho, \tau)$ are stretched space and time variables, respectively, corresponding to a strictly two-dimensional evolution; $\exp (a t) f_{n}(\rho)$ is the $\theta$-averaged vorticity for the spiral and $\exp (a t) g(\rho)$ specifies some axisymmetric background vorticity field.

We will later consider in detail the special case where $f_{n}(\rho)=f(\rho), n=0,1, \ldots$, in which case the spiral takes the form of a thin shear layer in the process of roll-up. In the inviscid limit, the spiral shape and vorticity distribution are given, respectively, by

$$
\begin{aligned}
& \theta=\Omega(\rho) \tau, \\
& \hat{\omega}(\rho, \theta, \tau)=2 \pi f(\rho) \delta[\theta-\Omega(\rho) \tau]+g(\rho),
\end{aligned}
$$

where $\delta$ is the Dirac delta function.

$L$ also calculates the spectrum produced by a random superposition of vortices with any specified core vorticity distribution. It is shown that an average over a shell of constant $k$ in wave-number space gives, for the velocity (energy) spectrum ( $L$ Sec. III B), 


$$
\begin{aligned}
& E(k)=E_{0}(k)+E_{s}(k), \\
& E_{0}(k)=\frac{\pi N}{k} \int_{\tau_{1}}^{\tau_{2}} \hat{I}_{0}^{2}(k, \tau) d \tau, \\
& E_{s}(k)=\int_{\tau_{1}}^{\tau_{2}} \tilde{E}_{s}(k, \tau) d \tau, \\
& \vec{E}_{s}(k, \tau)=\frac{2 \pi N}{k} \sum_{n=1}^{\infty}\left|\hat{I}_{n}(k, \tau)\right|^{2}, \\
& \hat{I}_{n}(k, \tau)=\int_{0}^{\infty} J_{n}\left(\frac{k \rho}{\sqrt{1+a \tau}}\right) \hat{\omega}_{n}(\rho, \tau) \rho d \rho,
\end{aligned}
$$

where $\tau_{1}$ and $\tau_{2}$ are early and late cutoff times, respectively, whose values will generally be model dependent and $J_{n}$ is the Bessel function of the first kind of order $n$. Equations (10)-(14) are exact for straight vortices with vorticity independent of $z$. The spectrum separates into two components; $E_{0}(k)$ which is the axisymmetric part or core produced by the $\theta$-averaged vorticity field, for which we may put $\tau_{1}=0$, and $E_{s}(k)$ which arises from the $|n| \geqslant 1$ Fourier components and which is referred to as the spiral. Unless where specified we will suppress the $\left(\tau_{1}, \tau_{2}\right)$ dependence of $E_{0}, E_{s}$. We remark that the stretching strain $a$ appears only under the square root in Eqs. (10)-(13). If $a$ is set equal to zero the spectrum of a collection of either aligned vortices in twodimensions or unstretched vortices in three dimensions is obtained. PS (Appendix B) show that these are given by the same formula.

\section{B. Dissipation in the spiral}

PS calculated $\overline{\omega^{2 p}}$ vorticity moments given by

$$
\begin{aligned}
\overline{\omega^{2 p}}= & N \int_{0}^{2 \pi} \int_{0}^{\infty} \int_{\phi / a}^{\tau_{2}}(1+a \tau)^{2 p-1} \\
& \times\left(\sum_{n=-\infty}^{\infty} \hat{\omega}_{n}(\rho, \tau) \exp (i n \theta)\right)^{2 p} \rho d \theta d \rho d \tau, \\
& p \geqslant 1,
\end{aligned}
$$

where we define the parameter $\phi$ by $\tau_{1}=\phi / a$. The special case $p=1$ gives the dissipation $\epsilon=\epsilon_{0}+\epsilon_{s}=\nu \overline{\omega^{2}}$,

$$
\begin{aligned}
\epsilon_{0}+\epsilon_{s}= & 2 \pi \nu N \int_{0}^{\infty} \int_{\phi / a}^{\tau_{2}}(1+a \tau) \\
& \times\left(\hat{\omega}_{0}^{2}(\rho, \tau)+2 \sum_{n=1}^{\infty} \hat{\omega}_{n}^{2}(\rho, \tau)\right) \rho d \rho d \tau,
\end{aligned}
$$

where $\epsilon_{s}$, the dissipation in the spiral, corresponds to the $n$-sum term in Eq. (16). Expanding the $1+a \tau$ factor, using Eqs. (2)-(8) and changing variables using $u=n^{2} C \tau^{3}$ then gives for $\epsilon_{s}$,

$$
\begin{aligned}
\epsilon_{s}= & \frac{4 \pi \nu N}{3} \sum_{n=1}^{\infty} \int_{0}^{\infty} \int_{n^{2} C \phi^{3} / a^{3}}^{\infty}\left|f_{n}(\rho)\right|^{2}\left(C^{-1 / 3} n^{-2 / 3} u^{-2 / 3}\right. \\
& \left.+a C^{-2 / 3} n^{-4 / 3} u^{-1 / 3}\right) e^{-u} \rho d \rho d u,
\end{aligned}
$$

where $C=2 \nu \Lambda^{2}(\rho) / 3$ and where, anticipating that the integrals will converge at $\infty$ with our choice of $f$, we have put $\tau_{2}=\infty$.

We now distinguish two special cases:

(1) The $f_{n}$ retain an $n$ dependency, but the decay with large $n$ is such that the sum $\Sigma_{0}^{\infty} n^{-2 / 3}\left|f_{n}\right|^{2}$ is convergent uniformly in $\rho$.

(2) $f_{n}(\rho)=f(\rho), n=0, \cdots$ where the absence of a subscript indicates no $n$ dependence. This case was considered by PS, and is that discussed earlier in relation to Eq. (9).

We first consider case (1). Here the lower limit of integration with respect to $u$ in Eq. (17) may be set to zero with small error, and we obtain, to leading order

$$
\begin{aligned}
\epsilon_{s}= & \frac{4 \pi}{3}\left(\frac{3}{2}\right)^{1 / 3} \Gamma\left[\frac{1}{3}\right] N \nu^{2 / 3} \sum_{n=1}^{\infty} n^{-2 / 3} \int_{0}^{\infty} \frac{\left|f_{n}(\rho)\right|^{2} \rho d \rho}{|\Lambda(\rho)|^{2 / 3}} \\
& +\frac{4 \pi}{3}\left(\frac{3}{2}\right)^{2 / 3} \Gamma\left[\frac{2}{3}\right] N a \nu^{1 / 3} \sum_{n=1}^{\infty} n^{-4 / 3} \int_{0}^{\infty} \frac{\left|f_{n}(\rho)\right|^{2} \rho d \rho}{|\Lambda(\rho)|^{4 / 3}} .
\end{aligned}
$$

For case (2), convergence of the first sum in Eq. (17) requires retention of the lower limit of integration. Here Eq. (17) may be expanded as a series in powers of $\phi^{1 / 2}$ by first breaking up the range of the $u$ integration in the inner integral followed by repeated use of the Euler summation formula in the form (see Carrier et al. ${ }^{8}$ )

$$
\begin{aligned}
\sum_{n=1}^{\infty} q(n)= & \frac{1}{2}[q(1)+q(\infty)]+\int_{1}^{\infty} q(n) d n \\
& +\frac{B_{2}}{2}\left[q^{\prime}(\infty)-q^{\prime}(1)\right]+\cdots
\end{aligned}
$$

where $B_{2}=1 / 6$ is a Bernoulli number, and exchanging of limits of integration in the $n-u$ plane. To $O\left(\phi^{1 / 2}\right)$ this gives

$$
\begin{aligned}
\epsilon_{s}= & 4 \pi \phi^{-1 / 2}\left(\frac{3}{2}\right)^{1 / 2} \Gamma\left[\frac{1}{2}\right] N a^{1 / 2} \nu^{1 / 2} \int_{0}^{\infty} \frac{|f(\rho)|^{2} \rho d \rho}{|\Lambda(\rho)|} \\
& -\frac{4 \pi}{3}\left(\frac{3}{2}\right)^{1 / 3} \Gamma\left[\frac{1}{3}\right] N a \nu^{2 / 3} \int_{0}^{\infty} \frac{|f(\rho)|^{2} \rho d \rho}{|\Lambda(\rho)|^{2 / 3}} \\
& +\frac{4 \pi}{3}\left(\frac{3}{2}\right)^{2 / 3} \Gamma\left[\frac{2}{3}\right] N a \nu^{1 / 3} \int_{0}^{\infty} \frac{|f(\rho)|^{2} \rho d \rho}{|\Lambda(\rho)|^{4 / 3}} \sum_{n=1}^{\infty} n^{-4 / 3} \\
& -4 \pi \phi^{1 / 2}\left(\frac{3}{2}\right)^{1 / 2} \Gamma\left[\frac{1}{2}\right] N a^{1 / 2} \nu^{1 / 2} \int_{0}^{\infty} \frac{|f(\rho)|^{2} \rho d \rho}{|\Lambda(\rho)|},
\end{aligned}
$$

where $\Gamma[\cdots]$ denotes the gamma function. Note that $a \tau \gg 1$ has not been used. PS (Sec. VI C) obtained the first and third terms of Eq. (20). 
The spectrum of the spiral should satisfy the kinematic identity

$$
\overline{\overline{\omega_{s}^{2}}}=2 \int_{0}^{\infty} k^{2} E_{s}(k) d k,
$$

where $\omega_{s}^{2}$ again refers to the $n$-sum term in Eq. (16). When the $k^{-5 / 3}$ spectrum of $L$ is used in the right-hand side of Eq. (21), only the second term of Eq. (18) and the third term of Eq. (20) respectively, are obtained. Moreover the first term on the right-hand side of Eq. (20) is divergent when $\phi \rightarrow 0$, or equivalently $\tau_{1} \rightarrow 0$. Since for case (2), the solution given by Eqs. (3) $-(6)$ is asymptotic to a vortex sheet when $\tau_{1} \rightarrow 0$, this suggests that there exists a further contribution to $E_{s}(k)$ from the period $a \tau \leqslant 1$.

Substituting Eqs. (2)-(6) in Eqs. (12) and (13) using the stationary-phase evaluation of the $\hat{I}_{n}$ integrals as in $L$ (this requires $k R \gg 1$ ), and retaining all $a \tau$ dependencies gives $\tilde{E}_{s}(k, \tau)$ in Eq. (13) as

$$
\begin{aligned}
\tilde{E}_{s}(k, \tau)= & -\frac{2 \pi N}{k^{3}}(1+a \tau) \\
& \times \exp \left[-\frac{2 \nu k^{2} \tau}{3(1+a \tau)}\right] \sum_{n=1}^{\infty} \rho|f(\rho)|^{2} \frac{\Lambda(\rho)}{\Lambda^{\prime}(\rho)},
\end{aligned}
$$

where $\Lambda^{\prime}(\rho)=d \Lambda / d \rho$ and, for given $k, n$ and $\tau, \rho(n, \tau, k)$ is a solution of

$$
k+n \Lambda(\rho) \tau(1+a \tau)^{1 / 2}=0 .
$$

When Eq. (22) is used in Eq. (13) and the integral in $\tau$ is transformed to a $\rho$ integral using Eq. (23), then on letting $\tau_{2} \rightarrow \infty$

$$
\begin{aligned}
E_{s}(k)= & \frac{2 \pi N}{k^{3}} \sum_{n=1}^{\infty} \int_{\rho_{1}}^{\infty}\left|f_{n}(\rho)\right|^{2} \\
& \times \exp \left[-\frac{2 \nu k^{2} \tau}{3(1+a \tau)}\right] \frac{2 \tau(1+a \tau)^{2}}{2+3 a \tau} \rho d \rho,
\end{aligned}
$$

where $\rho_{1}=\rho\left(n, \tau_{1}, k\right)$ and where $\tau$ is now a function of $k, n$, and $\rho$ through Eq. (23). When $a \neq 0$, the range of integration in Eq. (24) may be split into two parts, $a \tau<1$ and $a \tau>1$. We shall refer to $a \tau<1$, when, for case (2), the shear layer is thickening and both the stretching of vortex lines and the lateral compression of the vortex structure is small, as the vortex-sheet epoch.

\section{The vortex sheet epoch: $a \tau<1$}

We denote the contribution to the spectrum from this range by $E_{s_{1}}$. Setting $a=0$ in Eq. (24)-(23) and using, from Eq. (23), $\tau=k / n|\Lambda(\rho)|$ leads to

$$
\begin{aligned}
E_{s_{1}}(k)= & \frac{2 \pi N}{k^{2}} \sum_{n=1}^{\infty} \frac{1}{n} \\
& \times \int_{\rho_{1}}^{\infty} \exp \left[-\frac{2 \nu k^{3}}{3 n|\Lambda(\rho)|}\right] \frac{\left|f_{n}(\rho)\right|^{2} \rho}{|\Lambda(\rho)|} d \rho .
\end{aligned}
$$

For case (1), we can put $\rho_{1} \rightarrow 0$ in Eq. (25), and so obtain an expression for $E_{s_{1}}(k)$. This fails for case (2), since the sum would then be divergent as $n \rightarrow \infty$. Approximating the $n$ sum by an integral in $(1, \infty)$ and exchanging the limits of integration in the $(n-p)$ plane then gives

$$
\begin{aligned}
E_{s_{1}}(k)= & \frac{2 \pi N}{k^{2}} \int_{0}^{\infty} \int_{1}^{k /|\Lambda(\rho)| \tau_{1}} \frac{1}{n} \\
& \times \exp \left[-\frac{2 \nu k^{3}}{3 n|\Lambda(\rho)|}\right] \frac{|f(\rho)|^{2} \rho}{|\Lambda(\rho)|} d \rho d n .
\end{aligned}
$$

On applying the transformation $u=k / n|\Lambda(\rho)|$ we obtain

$$
\begin{aligned}
E_{s_{1}}(k)= & \frac{2 \pi N}{k^{2}} \int_{0}^{\infty} \int_{\tau_{1}}^{k|\Lambda(\rho)|} \frac{1}{u} \\
& \times \exp \left(\frac{-2 k^{2} \nu u}{3}\right) \frac{|f(\rho)|^{2} \rho}{|\Lambda(\rho)|} d \rho d u,
\end{aligned}
$$

which may be expressed in the form

$$
\begin{aligned}
E_{s_{1}}(k)= & \frac{2 \pi N}{k^{2}} E_{1}\left(\frac{2 \phi k^{2} \nu}{3 a}\right) \int_{0}^{\infty} \frac{|f(\rho)|^{2} \rho d \rho}{|\Lambda(\rho)|} \\
& -\frac{2 \pi N}{k^{2}} \int_{k /|\Lambda(\rho)|}^{\infty} E_{1}\left(\frac{2 k^{3} \nu}{3|\Lambda(\rho)|}\right) \frac{|f(\rho)|^{2} \rho d \rho}{|\Lambda(\rho)|},
\end{aligned}
$$

where $E_{1}(z)$ is the exponential integral. Corrections from the Euler summation formula (19) give higher order terms in $\phi$ which have been omitted. When $\phi \rightarrow 0, E_{s_{1}}$ is divergent as $k^{-2} \log \phi$. Further, since Eq. (28) has been obtained by setting $a=0$ in Eq. (14) [note that the appearance of $a$ in Eq. (28) is parametric through the definition of $\tau_{1}$ ], then it must be the spectrum of the unstrained spiral.

\section{The $k^{-5 / 3}$ contribution: $a \tau>1$}

Neglecting terms of $O(1)$ in comparison to $O(a \tau)$ in Eq. (24) gives

$$
\begin{aligned}
E_{s_{2}}(k)= & \frac{4 \pi}{3} N a^{1 / 3} k^{-5 / 3} \\
& \times \exp \left[-\frac{2 \nu k^{2}}{3 a}\right] \sum_{n=1}^{\infty} n^{-4 / 3} \int_{0}^{\infty} \frac{\left|f_{n}(\rho)\right|^{2} \rho d \rho}{|\Lambda(\rho)|^{4 / 3}} \\
& -4 \pi N \phi^{1 / 2} k^{-2} \exp \left[-\frac{2 \nu k^{2}}{3 a}\right] \int_{0}^{\infty} \frac{\left|f_{n}(\rho)\right|^{2} \rho d \rho}{|\Lambda(\rho)|}
\end{aligned}
$$

where the first term is the $L k^{-5 / 3}$ spectrum, obtained by putting $\rho_{1}=0$, and the second term is an $O\left(\phi^{1 / 2}\right)$ correction which follows by retaining finite but small $\rho_{1}$ and using the Euler summation formula. To $O\left(\phi^{1 / 2}\right)$ the composite spiral spectrum is $E_{s}\left(k \mid \tau_{1}\right)=E_{s_{1}}\left(k \mid \tau_{1}\right)+E_{s_{2}}(k)$ from Eq. (28) and Eq. (29) for case 2, where for emphasis we have temporarily restored the dependence of the results on the early cutoff $\tau_{1}$, and Eq. (25) (with $\rho_{1}=0$ ) and Eq. (29) for case 1. When the composite $E_{s}(k)$ is used in the right-hand side of 
Eq. (21) multiplied by $\nu$, [Eq. (18); case 1] and [Eq. (20); case 2] are obtained. Hence Eq. (21) is satisfied selfconsistently.

Lundgren ${ }^{4}$ has shown that the $k^{-5 / 3}$ form can be obtained from the spectrum of the unstrained flow via a similarity transformation. Since we have presently shown that $E_{s}(k)$ consists of the full spectrum of the unstrained spiral $E_{s_{1}}(k)$ [to $\left.O\left(\phi^{0}\right)\right]$ plus the $k^{-5 / 3}$ term, this may seem paradoxical. There is no inconsistency, however, since the extra terms arise presently from a splitting of the range of integration in Eq. (14) which is only meaningful when $a \neq 0$.

\section{E. The axisymmetric component}

For the remainder of this paper we will consider only case 2, except in Sec. III, where we will temporarily restore the $n$ dependence of the $f_{n}$. We will later require the axisymmctric component of the spectrum at a finite upper cutoff time $\tau_{2}$. In order to make a specific calculation of $E_{0}\left(k \mid \tau_{1}, \tau_{2}\right)$ we introduce dimensionless forms of the shape functions $f(\rho), g(\rho), \Omega(\rho)$, and $\Lambda(\rho)$ in the form

$$
\begin{aligned}
& f(\rho)=\frac{\Gamma_{0}}{R^{2}} \tilde{f}(\xi), \quad g(\rho)=\frac{\Gamma_{0}}{R^{2}} \tilde{g}(\xi), \\
& \Omega(\rho)=\frac{\Gamma_{0}}{R^{2}} \tilde{\Omega}(\xi), \quad \Lambda(\rho)=\frac{\Gamma_{0}}{R^{3}} \tilde{\Lambda}(\xi),
\end{aligned}
$$

where $\xi=\rho / R, \Gamma_{0}$ is a circulation scale and $R$ is the lateral scale of the vortex structure. Following PS we make a choice of $\tilde{f}(\xi), \tilde{g}(\xi)$ as

$$
\begin{aligned}
& \tilde{f}(\xi)=\frac{1}{\pi \Gamma[1+\alpha / 2]} \xi^{\alpha} e^{-\xi^{2}}, \\
& \tilde{g}(\xi)=-\frac{1}{\pi} \xi^{2} e^{-\xi^{2}} .
\end{aligned}
$$

In Eq. (32) $\alpha$ is a parameter with values in the range $0>\alpha \geqslant-1$. It is easily verified that $\tilde{\Lambda}<0$ as required. For the choice $\alpha=-0.5$ the radial circulation distribution shows a maxima near $\xi=0.945$ where $\Gamma(0.95) \approx 0.480 \Gamma_{0}$.

The axisymmetric spectrum component can estimated for large $k$ from knowledge of the evolution of the zeroth order component of Eq. (3), which we denote by $\omega_{0}(r, t)$. In PS this was obtained as a solution of the "stretched" heat equation, and is given by

$$
\begin{aligned}
\omega_{0}(r, t)= & \frac{e^{a t}}{2 \nu \tau(t)} \exp \left(\frac{-r^{2} e^{a t}}{4 \nu \tau}\right) \int_{0}^{\infty}[f(u)+g(u)] \\
& \times \exp \left(-\frac{u^{2}}{4 \nu \tau(t)}\right) I_{0}\left[\frac{r e^{a t / 2} u}{2 \nu \tau(t)}\right] u d u,
\end{aligned}
$$

where $\tau(t)$ is given by the second of Eq. (6) and $I_{0}$ is the modified Bessel function. It is easily verified from $\mathrm{Eq}$. (34) that the total circulation in the vortex

$$
\Gamma(\infty)=2 \pi \int_{0}^{\infty} \omega_{0}(r, t) r d r=2 \pi \int_{0}^{\infty}[f(u)+g(u)] u d u,
$$

is an invariant of the evolution. The choice (32)-(33) gives $\Gamma(\infty)=0$. If Eq. (33) is replaced by $\tilde{g}(\xi)=0$, we have a model with finite circulation at infinity $\Gamma(\infty)=I_{0}$. We consider presently the case $\Gamma(\infty)=0$. Substituting Eqs. (27)-(32) into Eq. (34) and using the result in Eq. (14) then leads to

$$
\begin{aligned}
\hat{I}_{0}\left(k, \mid \tau_{1}, \tau_{2}\right)= & \Gamma_{0} \exp \left(-\frac{\nu \tau k^{2}}{1+a \tau}\right) H(k R, \alpha ; a \tau), \\
H(k R, \alpha ; a \tau)= & \frac{1}{2 \pi} \exp \left(-\frac{k^{2} R^{2}}{4(1+a \tau)}\right)\left[\frac{k^{2} R^{2}}{4(1+a \tau)}-1\right. \\
& \left.+M\left(-\frac{\alpha}{2}, 1, \frac{k^{2} R^{2}}{4(1+a \tau)}\right)\right],
\end{aligned}
$$

where $M$ is the confluent hypergeometric function. Making the approximation $a \tau \gg 1$ in the exponential of Eq. (36) and using the transformation $v(\tau, k R)=k^{2} R^{2} / 4(1+a \tau)$ we can obtain, using Eq. (11)

$$
\begin{aligned}
E_{0}\left(k \mid \tau_{1}, \tau_{2}\right)= & N R^{2} \Gamma_{0}^{2} a^{-1 / 2} \nu^{-1 / 2} K \\
& \times \exp \left(-K^{2} / 2\right) A\left(\alpha, \nu_{1}, \nu_{2}\right), \\
A\left(\alpha, \nu_{2}, \nu_{1}\right)= & \frac{1}{32 \pi} \int_{v_{2}}^{v_{1}} e^{-2 v}[v-1 \\
& \left.+M\left(-\frac{\alpha}{2}, 1, v\right)\right]^{2} \frac{1}{v^{2}} d v,
\end{aligned}
$$

where $K=2 k(\nu / a)^{1 / 2}, v_{1}=v\left(\tau_{1}, k R\right), v_{2}=v\left(\tau_{2}, k R\right)$. If $k R$ is large and $a \tau_{1}$ is $O(1)$, then the upper limit can be set to $\infty$ with small error. The axisymmetric spectrum used by PS is recovered from Eq. (38) when $\tau_{2}$ is set to $\infty$. If $\Gamma(\infty)$ is nonzero, then the axisymmetric core may be shown to be asymptotic, for large $\tau$, to the equilibrium Burgers vortex. The leading order axisymmetric spectrum is then of the form $\tau_{2} k^{-1} \exp \left(-2 k^{2} \nu / a\right)$ in agreement with Townsend ${ }^{y}$ (see Sec. VI for further discussion of this case).

In terms of the parameters of Eq. (38), the $L k^{-5 / 3} \mathrm{spec}$ trum [leading term of Eq. (29)] is

$$
\begin{aligned}
& \left.E_{s}(k)=D_{0}(\alpha) N R^{2} \Gamma_{0}^{2 / 3} a^{-1 / 2} \nu^{5 / 6} K^{-5 / 3} \exp \left[-K^{2} / 6\right)\right], \\
& D_{0}(\alpha)=\frac{2^{11 / 3} \pi}{3} \int_{0}^{\infty} \frac{\tilde{f}^{2}(\xi)}{|\tilde{\Lambda}(\xi)|^{4 / 3}} \xi d \xi \sum_{1}^{\infty} n^{-4 / 3}
\end{aligned}
$$

\section{SPECTRA OF $\omega^{2}$ AND $\epsilon$}

PS Eq. (B16) show that the power spectrum of the product of two arbitrary scalar-invariant functions of position in the vortex structures $\mathscr{F}\left(r_{1}, r_{2}\right), \quad \mathscr{r}\left(r_{1}, r_{2}\right)$, where $r_{1}=r \cos \theta, r_{2}=r \sin \theta$, can be expressed as

$$
E_{f g}(k)=4 \pi^{2} N k \int_{\tau_{1}}^{\tau_{2}} \oint \hat{\mathscr{F}}\left(k_{1}, k_{2}\right) \hat{\zeta}^{*}\left(k_{1}, k_{2}\right) d \tau d \theta_{k},
$$

where $\hat{\mathscr{F}}\left(k_{1}, k_{2}\right), \hat{\mathscr{G}}\left(k_{1}, k_{2}\right)$ are sectional Fourier transforms, $k_{1}=k \cos \theta_{k}, k_{2}=k \sin \theta_{k}$, and ${ }^{*}$ denotes the complex conjugate. The spectrum of the dissipation, given pointwise within vortex structures by 


$$
\epsilon=2 \nu\left(e_{1}^{2}+e_{2}^{2}+e_{3}^{2}\right),
$$

where $\left(e_{1}, e_{2}, e_{3}\right)$ are the local principal rates of strain within a vortex structure, can be calculated by choosing $F=F=\epsilon$. PS also show (Sec. VB) that within the spiral structure the principal rates of strain associated with the nonaxisymmetric spiral modes are given by, to leading order,

$$
e_{1}=-\frac{1}{2} a+\frac{1}{2} \omega^{\prime}, \quad e_{2}=-\frac{1}{2} a-\omega^{\prime}, \quad e_{3}=a,
$$

where the prime denotes that the $n-0$ term is omitted. As will be demonstrated in Sec. $V$, the dissipation associated with the axisymmetric field is small compared to that contributed by the nonaxisymmetric modes. From Eqs. (43) and (44) it follows that $\epsilon=\nu \omega^{\prime 2}+3 \nu a^{2}$, and, since it will be seen that $a^{2} / \overline{\omega^{2}} \ll 1$, then to a good approximation $\epsilon=\nu \omega^{2}$ pointwise for the spiral vortex. Therefore, the spectra of $\epsilon$ and of $\omega^{2}$ are related as

$$
E_{\epsilon \epsilon}(k) \approx \nu^{2} E_{\omega^{2} \omega^{2} \omega^{2}}(k)
$$

and we note that this result is model dependent.

We estimate $E_{\omega^{2} \omega^{2}}(k)$ by first writing, in $(\rho-\tau)$ coordinates

$$
\begin{aligned}
& \hat{\omega}^{2}=\sum_{-\infty}^{\infty} \hat{A}_{n} e^{i n \theta}, \\
& \hat{A}_{n}=\sum_{m=-\infty}^{m=\infty} \hat{\omega}_{m} \hat{\omega}_{n-m} .
\end{aligned}
$$

By following the steps leading to Eqs. (10)-(11), but now with $\omega$ replaced by $\omega^{2}$, the shell-summed spectrum of $\omega^{2}$ may then be shown to be given approximately by

$E_{\omega^{2} \omega^{2}}(k)=2 \pi N k \int_{\tau_{1}}^{\tau_{2}}(1+a \tau)^{2}\left(\left|\hat{B}_{0}\right|^{2}+2 \sum_{n=1}^{\infty}\left|\hat{B}_{n}\right|^{2}\right) d \tau$

$\hat{B}_{n}(k, \tau)=\int_{0}^{\infty} J_{n}\left(\frac{k \rho}{\sqrt{1+a \tau}}\right) \hat{A}_{n}(\rho, \tau) \rho d \rho$,

where from Eqs. (4) and (47)

$\hat{A}_{n}=e^{-i n \Omega(\rho) \tau} \sum_{m=-\infty}^{\infty} f_{m}(\rho) f_{n-m}(\rho) e^{-\left[m^{2}+(n-m)^{2}\right] \nu \tau^{3} \Lambda^{2}(\rho) / 3}$,

where we have temporarily restored the $n$ dependence of the $f_{n}$. Using Eq. (50) in Eq. (49), estimating the integral using stationary phase and putting $\tau_{2}=\infty$ in Eq. (48) gives, for $E_{\omega^{2} \omega^{2}}$

$$
\begin{aligned}
E_{\omega^{2} \omega^{2}}(k)= & \frac{8 \pi N}{k} \sum_{n=1}^{\infty} \int_{\rho_{1}}^{\infty}\left(\sum_{m=-\infty}^{\infty} f_{m}(\rho) f_{n-m}(\rho)\right. \\
& \left.\times \exp \left(-\frac{\left[m^{2}+(n-m)^{2}\right] \nu k^{2} \tau}{3(1+a \tau) n^{2}}\right)\right)^{2} \\
& \times \frac{\tau(1+a \tau)^{4}}{2+3 a \tau} \rho d \rho
\end{aligned}
$$

where Eq. (23) and the discussion following this equation applies. In order to proceed further it appears necessary to introduce assumptions concerning the $n$ dependence of the $f_{n}$, and we therefore again specialize to case 2 . We then make the approximation

$$
\frac{a \tau(1+a \tau)^{4}}{2+3 a \tau} \approx \frac{1}{3} a \tau(1+a \tau)^{3}
$$

with small relative error over the range of large $a \tau$ which contributes most to the integral, put $a \tau \gg 1$ in the exponential and approximate the $m$ sum by an integral. Expanding the $(1+a \tau)^{3}$ factor and using Eq. (23) with $a \tau \gg 1$ to eliminate $\tau$, we obtain, after some algebra

$$
\begin{aligned}
E_{\omega^{2} \omega^{2}}(k)= & \frac{4 \pi^{2} N}{\nu} \exp \left(\frac{-k^{2} \nu}{3 a}\right) \sum_{q=1}^{4} Q_{q} k^{2 q / 3-3} a^{q-q / 3} \\
& \times \sum_{n=1}^{\infty} n^{2-2 q / 3} \int_{\rho_{1}}^{\infty} \frac{|f(\rho)|^{4} \rho d \rho}{|\Lambda(\rho)|^{2 q / 3}}
\end{aligned}
$$

where $Q_{1}=Q_{4}=1, Q_{2}=Q_{3}=3$. We remark that use of $a \tau \gg 1$ in Eq. (23) is not strictly valid for the $q=1$ term in Eq. (52) which arises from the $O(1)$ contribution in the $(1+a \tau)^{3}$ expansion; use of $k+n \Lambda \tau=0$ for this term gives a slightly different result for $q=1$ in Eq. (52). The present approximation will be justified post hoc.

As with Eq. (25) we cannot now put $\rho_{1}=0$ since this would produce a divergent series. We thus again approximate the $n$ sum by an integral and exchange the limits of integration in the $(n-\rho)$ plane. Performing the $n$ integral then pulls down powers of $k$ that just cancel the $k^{2 q / 3-3}$ factor in each term of the $q$ sum of Eq. (52), to give, on putting $\tau_{1}=\phi / a$,

$$
\begin{aligned}
E_{\omega^{2} \omega^{2}}(k)= & 12 \pi^{2} N a^{3} \nu^{-1} \exp \left(\frac{-k^{2} \nu}{3 a}\right) \int_{0}^{\infty} \frac{|f(\rho)|^{4} \rho d \rho}{|\Lambda(\rho)|^{3}} \\
& \times \sum_{q=1}^{4} \frac{Q_{q}}{(9-2 q) \phi^{9 / 2-q}}
\end{aligned}
$$

The shell-summed spectrum of $\epsilon$ follows from Eq. (45). We comment that Eq. (53) is independent of the probability distributions ( $\mathrm{pdfs}$ ) of the orientations of the vortex structures with respect to laboratory axes, and so does not depend on statistical isotropy. The result does, however, depend on the assumed $n$ independence of the $f_{n}$. When the right-hand side of Eq. (53) is integrated over all $k$, the resulting expression for $\omega^{4}$ is in exact agreement with expressions for this quantity given by PS $(82 a)-(82 b)$ [see also the present Appendix $\mathrm{B}$, Eq. (B3)]. This supports the validity of the approximations used in obtaining Eq. (53).

If statistical isotropy is assumed, that is, the pdfs of the orientations of the structures are assumed uniform on the unit sphere, then the one-dimensional spectra can be obtained from standard results. This gives, for the one-dimensional dissipation spectrum $E_{\epsilon \epsilon}^{(1)}\left(k_{1}\right)$ 


$$
\begin{aligned}
E_{\epsilon \epsilon}^{(1)}(k)= & 3 \pi^{2} N a^{3} \nu E_{1}\left[\frac{k_{1}^{2} \nu}{3 a}\right] \int_{0}^{\infty} \frac{|f(\rho)|^{4} \rho d \rho}{|\Lambda(\rho)|^{3}} \\
& \times \sum_{q=1}^{4} \frac{Q_{q}}{(9-2 q) \phi^{9 / 2-q}}
\end{aligned}
$$

where $E_{1}$ is the exponential integral. When $k_{1}^{2} \nu / 3 a \ll 1$, Eq. (54) shows that $E_{\epsilon \epsilon}^{(1)}\left(k_{1}\right) \sim-\log \left(k_{1}\right)$. There is no inverse power-law range of either the shell-summed or the onedimensional spectra. Tsinober et al. ${ }^{10}$ used a 12-wire hotwire probe to measure three velocity components and their 9 gradients in turbulent grid flow at Taylor Reynolds number $R_{\lambda}$ in the range 60-90. The measured $E_{\varepsilon \epsilon}^{(1)}\left(k_{1}\right)$ shown in their Fig. 9(a) does not appear to exhibit an inverse powerlaw range at wave numbers below the dissipation range.

\section{A NUMERICAL TEST}

\section{A. Numerical evaluation of the spectrum integrals}

There have been several attempts to search for a $k^{-5 / 3}$ spectrum using numerical simulations of vortex roll-up. Buntine and Pullin ${ }^{11}$ studied two and several ${ }^{12}$ vortices merging in the presence of an axisymmetric strain field. They solved the Navier-Stokes equations with Burgers-like vortices as initial conditions and calculated the spectrum via the numerical evaluation of the $\hat{I}_{n}$ integrals. Their maximum $\Gamma / \nu$ was of order $10^{4}$ and their effective $R^{2} a / 4 \nu$ was $O(1)$. They found no inertial range. Lundgren ${ }^{4}$ performed several twodimensional calculations and used a similarity transformation of the two-dimensional vorticity spectrum to obtain the energy spectrum for an equivalent strained vortex. In one case spectral solutions of the Navier-Stokes equations in a doubly periodic geometry were obtained using a specific model of Eqs. (2) -(5) as initial conditions. The results were compared to those found using a numerical implementation of an analytical spiral. In a second calculation Navier-Stokes solutions were obtained using as an initial condition a large vortex surrounded by ten small vortices. Values of $\Gamma / \nu$ in the range $10^{4}-10^{5}$ were used, and in terms of our notation the effective $R^{2} a / 4 \nu$ was $O(\Gamma / \nu)$. For each flow a small $k^{-5 / 3}$ range was found.

The purpose of the present numerical tests is to estimate the range of validity of the original analytical result given in $L$ and to evaluate the magnitudes of the corrections found presently. In all calculations reported here we utilize the vorticity distribution given by the asymptotic form (2)-(8) of the Lundgren stretched-spiral with the functions $f$ and $g$ given by Eq. (30) and Eqs. (32)-(33). The numerical calculation of $E_{s}(k)$ first requires calculation of the Besselfunction integrals (14) and evaluation of the sum in Eq. (13) to give $\tilde{E}_{s}(k, \tau)$, followed by numerical evaluation of the $\tau$ integral (12). We will discuss two separate numerical procedures. In the first, which we refer to as the numerical double integral method, both the Bessel-function integrals and the $\tau$ integral are performed numerically. In the second, referred to as the stationary-phase-numerical method, the Besselfunction integrals are calculated by the $L$-stationary-phase

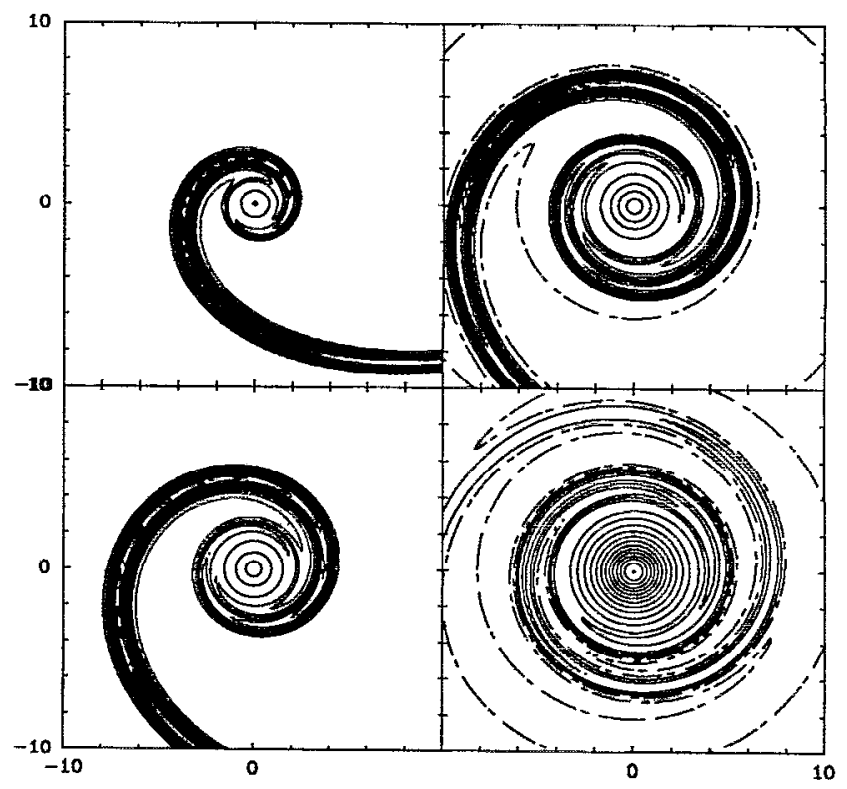

FIG. 1. Vorticity contours for spiral. $\alpha=-0.5, \Gamma_{n} / \nu=10^{4},\left(R^{2} a / 4 \nu\right)^{1 / 2}=20$. $x, y$ scaled against $2(\nu / a)^{1 / 2}$. Times at top to bottom, left to right; $a t=0.5$, $1.0,1.5,2.5 .-, \omega>0,----, \omega<0$.

asymptotics, and $\tilde{E}_{s}(k, \tau)$ is calculated from Eq. (22)-(23). At fixed $k$ the $\tau$ integral is then calculated numerically.

\section{B. Numerical double integral method}

The vorticity components $\hat{\omega}_{n}, n \neq 0$ were calculated directly from Eq. (4). The Bessel-function integrals were computed by $M$-point numerical integration using decomposition of the range of integration based on a table of zeroes for each order, and for most calculations $M=512$. The series (13) was then summed to $M$ terms at each $k$ and $\tau$. Finally, at fixed $k$ the $\tau$ integration was performed by Simpsons rule to give $E_{s}(k)$. The axisymmetric vorticity field $\omega(\rho, \tau)$ was found from numerical integration of the stretched heat equation PS(29) from the singular initial condition (30)-(33). The Bessel-function integration and $\tau$ integration to give $E_{0}(k)$ were treated as for the nonaxisymmetric component. For nondimensionalization we set $a=4, \nu=1$, which gives $k \equiv K$, and for purposes of comparison with the asymptotic results we put $N=1$. The independent parameters are then $\alpha, \Gamma_{0} / \nu$, $a R^{2} / 4 \nu$ and the initial time which we characterize by $\phi$ where, using Eq. (5), $a t_{1}=\log \left(1+a \tau_{1}\right)$ and we have previously set $\tau_{1}=\phi / a$. For all cases the time integration was continued to $a t_{2}=\log \left(1+a \tau_{2}\right)$ sufficiently large to ensure that the integrals (11) and (13) had converged. This generally required $a t_{2} \sim O(10)$ or larger, i.e., $a \tau_{2}=O\left(10^{4}\right)$. Calculations reported presently were done with $\alpha=-0.5$ and $\phi=0.475$, the values used by PS. Their Table 1 shows $D_{0}(-0.5)=7.03$ (see also present Table VI). Values of Reynolds number and vortex radius used were $\Gamma_{0} / \nu=10^{2}$, $10^{3}, 10^{4}$ and $\left(R^{2} a / 4 \nu\right)^{1 / 2}=5,20,50$.

Figure 1 shows four snapshots of the vorticity distribution at different values of at during the evolution with $\Gamma_{0} / \nu=10^{4}$ and $\left(R^{2} a / 4 \nu\right)^{1 / 2}=20$. At each $t(\tau)$ the nonaxisymmetric component was computed by calculation of the 


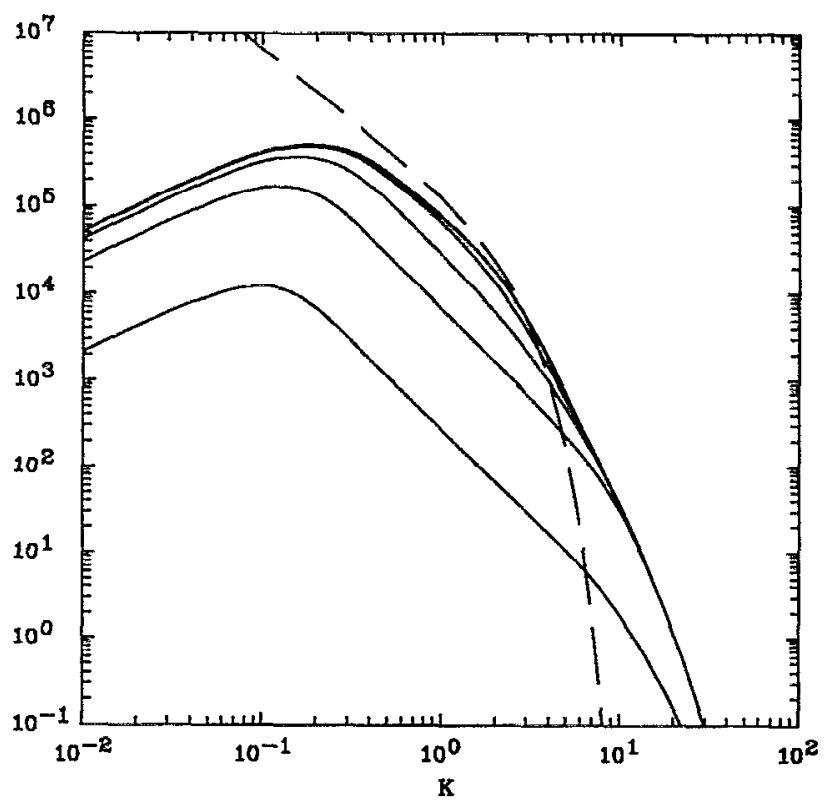

FIG. 2. Cumulative nonaxisymmetric energy spectrum $E_{s}(K)$, $K=2 k(\nu / a)^{1 / 2} \cdot \alpha=-0.5, \Gamma_{n} / \nu=10^{3},\left(R^{2} a / 4 \nu\right)^{1 / 2}=20, \phi=0.475 .$, , Numerical double-integral method. Times $a t_{2}\left(a \tau_{2}\right), 0.51(0.67), 1.5(3.48)$, 2.5(11.2), 3.5(32.1), 5.01(148.9), 5.5(243.70); last two indistinguishable. ---, Lundgren $k^{-5 / 3}$ asymptotic.

sum in Eq. (3) from $-M / 2$ to $M / 2-1$, and this was then added to $\omega_{0}(r, \tau)$. As the spiral rolls up, the vorticity is swept inwards by the radial component of the external strain and the distance between turns reduces rapidly due to both differential rotation and compression. This is the mechanism of the cascade (see Appendix A for further discussion), in agreement with the temporal shift of enstrophy to higher wave numbers suggested by Lundgren. ${ }^{4}$ In Fig. 2 the cumulative nonaxisymmetric spectrum [i.e., the numerically calculated value of the integral (12)] is plotted at a sequence of values of the upper cutoff time $\tau_{2}$, for $\Gamma_{0} / \nu=10^{3}$ and $\left(R^{2} a / 4 \nu\right)^{1 / 2}=20$. At early times a $K^{-2}$ form extending over several decades is clearly visible, followed later by the formation of a short $K^{-5 / 3}$ range as the spectrum of the spiral modes converges. Figure 3 shows the large-time converged spectra $E_{0}(K)$ and $E_{s}(K)$ compared with Eq. (38) for $E_{0}(K)$, in the limit $a \tau_{2} \rightarrow \infty$ and with the $L k^{-5 / 3}$ form (40) for the nonaxisymmetric asymptotic spectrum. At low $K$ the numerical results show a $K$ range in $E_{s}(K)$. It seems clear from Fig. 3(b) that the asymptotics for the nonaxisymmetric spectrum does not correctly capture the dissipation range spectrum and somewhat overestimates the inertial range.

Figure 4 shows the calculated converged nonaxisymmet ric spectra plotted in the form $K^{5 / 3} E_{s}(K)$ and compared with Eq. (40) for several combinations of $\Gamma_{0} / \nu$ and $R^{2} a / 4 \nu$. With $\Gamma_{0} / \nu=100$, the value used by PS, there is no identifiable $K^{5 / 3}$ region. When $\Gamma_{0} / \nu=10^{3}$ a plateau in the calculated $K^{5 / 3} E_{s}(K)$ can be seen. This becomes more extended as $\Gamma_{0} / \nu$ is increased with $\left(a R^{2} / 4 \nu\right)^{1 / 2}=20$ and 50 .

Table I shows the calculated dissipation of the spiral modes. The fourth and fifth columns show this quantity calculated from $\nu \Sigma_{n \neq 0} \bar{\omega}_{n}^{2}$ and $2 \nu \int_{0}^{\infty} k^{2} E_{s}(k) d k$, respectively. Since these should agree, differences, which are $2 \%-5 \%$, may be interpreted as a diagnostic for the accuracy of the spectrum calculation. The sixth and seventh columns show the spiral dissipation calculated, respectively, from the $L$ $k^{-5 / 3}$ asymptotics, given by the third term of Eq. (20), and from the sum of all terms on the right-hand side of Eq. (20). It is evident that, for the range of parameters surveyed Eq. (20) does not provide a better estimate than the single term $L$ $k^{-5 / 3}$ result, and in one case, $\Gamma / \nu=10^{4}, a R^{2} /(4 \nu)=5$, the complete Eq. (20) gives a negative dissipation. This suggests (a)

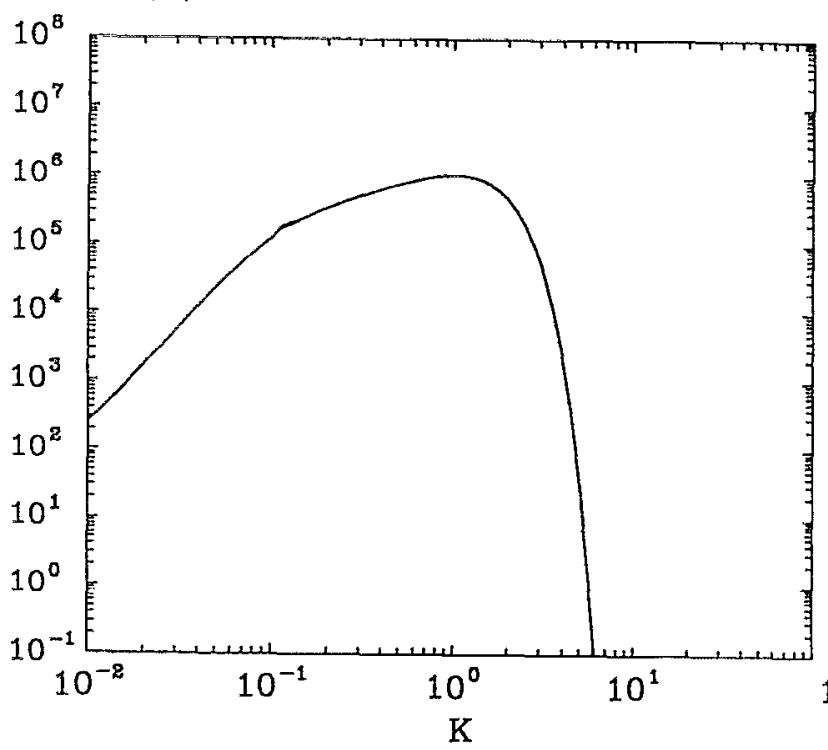

(b)

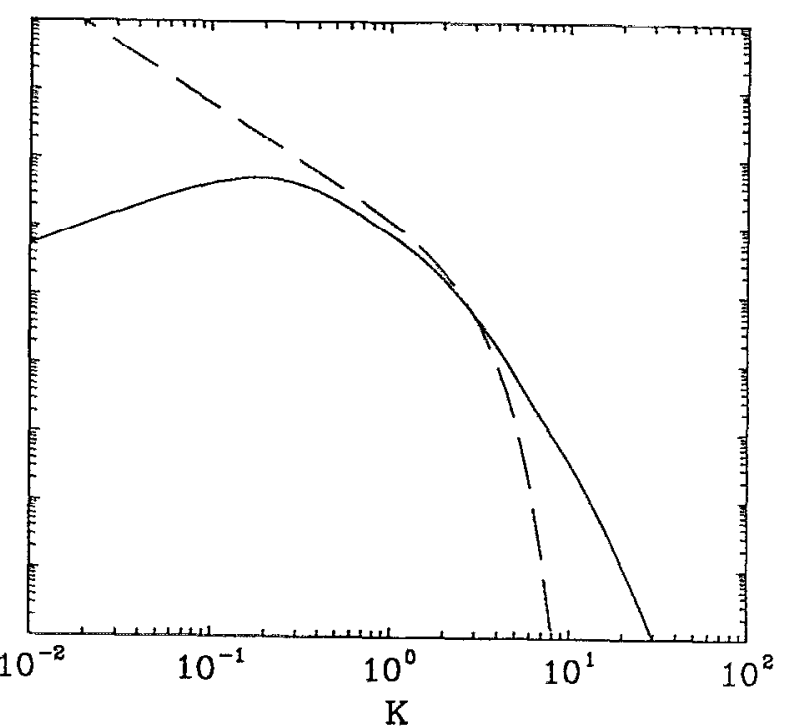

FIG. 3. Time integrated energy spectra vs $K, \alpha=-0.5, \Gamma_{0} / \nu=10^{3},\left(R^{2} a / 4 \nu\right)^{1 / 2}=20, \phi=0.475$. (a) Axisymmetric $E_{0}(K)$ :-numerical solution of heat equation. (b) Nonaxisymmetric $E_{s}(K):-$, numerical.---, Lundgren $k^{-5 / 3}$ asymptotic. Time $a t_{2}=12.1, a \tau_{2}=1.79 \times 10^{5}$. 


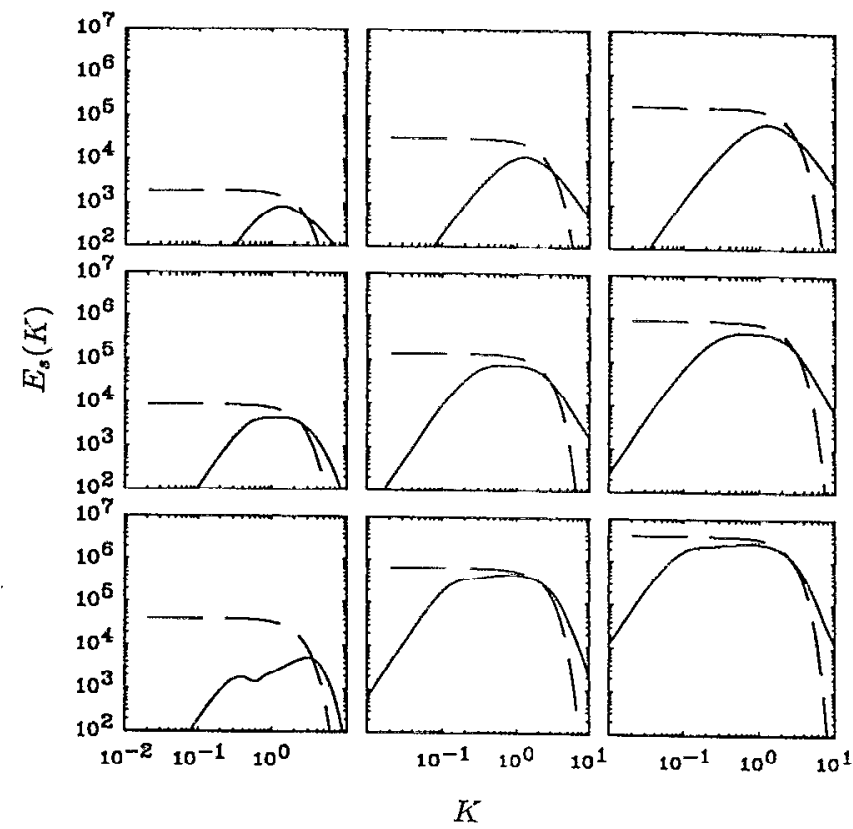

FIG. 4. Time integrated nonaxisymmetric energy spectrum in form $K^{5 / 3} E_{5}(K)$ vs $K . \alpha=-0.5$. Top $\Gamma_{0} / \nu=10^{2}$, middle $\Gamma_{0} / \nu-10^{3}$, bottom $\Gamma_{i} / \nu=10^{4}$. Left $\left(R^{2} a / 4 \nu\right)^{1 / 2}=5$, middle $\left(R^{2} a / 4 \nu\right)^{1 / 2}=20$. Right $\left(R^{2} a / 4 \nu\right)^{1 / 2}=50,-$, numerical double-integral method. ---, Lundgren $k^{-5 / 3}$.

that the series in $\phi$ for which Eq. (20) gives the first few terms is asymptotic rather than convergent, and that the $L$ $k^{-5 / 3}$ spectrum may be taken as a valid leading order approximation provided $\phi=O(1)$. We comment that the $L$ $k^{-5 / 3}$ asymptotics gives a good approximation to the dissipation over the range of parameters surveyed despite the apparent inaccuracies in the upper dissipation range spectrum. We again emphasize that Eq. (20) does not depend on either $a \tau \gg 1$ or on stationary phase evaluation of integrals.

The third column of Table I gives the dissipation for the fully converged axisymmetric mode, and since three-four figure agreement was obtained between the computed $\nu \overline{\omega_{0}^{2}}$ and $2 \nu \int_{0}^{\infty} k^{2} E_{0}(k) d k$, we tabulate only the former. When Eq. (38) with $\tau_{2}=\infty$ is used to compute the dissipation with $\Gamma_{0} / \nu=10^{3}$ we find values showing three-figure agreement with Table I. When $\Gamma_{0} / \nu=10^{2}$, Table I shows that the dissipation in the nonaxisymmetric modes exceeds that of the converged axisymmetric mode but this is reversed at $\Gamma_{0} / \nu=10^{3}, 10^{4}$. We stress that this true is for $\tau_{2}=\infty$ but not for the model with finite $\tau_{2}$ to be discussed in Sec. V.

\section{Stationary-phase-numerical method}

For this calculation $\tilde{E}_{5}(k, \tau)$ is computed from Eq. (22). In order to evaluate the sum with $k$ and $\tau$ fixed, Eq. (23) must be solved numerically for $\rho(n, k, \tau)$ for each $n, n=1, \ldots$, $n_{\max }$. This was done by a Newton-Raphson method. The functions of $\rho(n, k, \tau)$ under the summation can then be calculated and the sum to $n_{\max }$ performed. Presently $n_{\max }=2048$ was used. The time integral was then done at each $k$ using Simpsons rule.

In Fig. 5 we compare $\tilde{E}_{s}(K, a t)$ computed using the two methods described above at various at for $\Gamma_{0} / \nu=10^{3}$ and $\left(a R^{2} / 4 \nu\right)^{1 / 2}=20$. This figure can be considered as a test of the accuracy of the sum over $n$ of the Bessel-function integrals evaluated by stationary phase. For the range of at which contributes most to the time integral, the comparison is good but not excellent for $k R \gg 1$. A notable feature of the stationary-phase results is that there is a definite transition from a near- $K^{-1}$ behavior to a $K^{-2}$ behavior at a value of $K$ which increases with increasing at. Figure 6 shows the timeintegrated nonaxisymmetric spectrum $E_{s}(K)$ calculated by the stationary-phase-numerical method and compared with the $L$ asymptotic result. There is very good agreement in the $K^{-5 / 3}$ range, indicating that in the time integral, the weighting of the $K^{-1}$ and $K^{-2}$ ranges of $\tilde{E}_{s}(K, a t)$ is such that a $K^{-5 / 3}$ time-averaged spectrum results. In the dissipation range the numerical evaluation of the time integral gives a spectrum which substantially exceeds the asymptotic $\exp \left(-2 k^{2} \nu / 3 a\right)$ form. This apparently comes from the time integration. In Fig. 7 both sets of calculations show that the instantaneous spectrum in the dissipation range is approximately of the $\exp \left[-2 \nu k^{2} \tau / 3(1+a \tau)\right]$ form. Thus roll-off occurs at different $K$ for different at. When integrated over time this shift then gives rise to an $E_{s}(K)$ with slower than $\exp \left(-2 \nu k^{2} / 3 a\right)$ roll-off. In the asymptotics leading from Eq. (24) to Eq. (29), however, the approximation $\tau /(1+a \tau)$ $\approx 1 / a$ is made in the exponential, and this seems to lead to inaccuracies in the dissipation range arising from contribu-

TABI.F. I. Dissipation from axisymmetric vorticity, and dissipation calculated from vorticity field and from energy spectrum for nonaxisymmetric field. Numerical and asymptotic results. $\phi=0.475$.

\begin{tabular}{|c|c|c|c|c|c|c|}
\hline \multirow{2}{*}{$\frac{\Gamma_{0}}{\nu}$} & \multirow{2}{*}{$\frac{u R^{2}}{4 \nu}$} & \multicolumn{3}{|c|}{ Numerical } & \multicolumn{2}{|c|}{ Asymptotic } \\
\hline & & $\nu \overline{\omega_{0}^{2}}$ & $\nu \overline{\omega_{n}^{2}}$ & $2 \nu \int_{0}^{\infty} k^{2} E_{s}(k) d k$ & Lundgren $^{a} k^{-5 / 3}$ & Eq. (18) \\
\hline $10^{2}$ & 5 & $4.48 \times 10^{3}$ & $9.25 \times 10^{3}$ & $9.08 \times 10^{3}$ & $8.46 \times 10^{3}$ & $9.26 \times 10^{3}$ \\
\hline $10^{2}$ & 20 & $6.88 \times 10^{4}$ & $1.23 \times 10^{5}$ & $1.18 \times 10^{5}$ & $1.35 \times 10^{5}$ & $1.42 \times 10^{5}$ \\
\hline $10^{2}$ & 50 & $4.21 \times 10^{5}$ & $7.42 \times 10^{5}$ & $7.10 \times 10^{5}$ & $8.46 \times 10^{5}$ & $8.65 \times 10^{5}$ \\
\hline $10^{3}$ & 5 & $4.48 \times 10^{5}$ & $3.82 \times 10^{4}$ & $3.81 \times 10^{4}$ & $3.93 \times 10^{4}$ & $3.37 \times 10^{4}$ \\
\hline $10^{3}$ & 20 & $6.88 \times 10^{6}$ & $6.40 \times 10^{5}$ & $6.06 \times 10^{5}$ & $6.28 \times 10^{5}$ & $6.81 \times 10^{5}$ \\
\hline $10^{3}$ & 50 & $4.21 \times 10^{7}$ & $3.54 \times 10^{6}$ & $3.37 \times 10^{6}$ & $3.92 \times 10^{6}$ & $4.09 \times 10^{6}$ \\
\hline $10^{4}$ & 5 & $4.48 \times 10^{7}$ & $6.16 \times 10^{4}$ & $6.14 \times 10^{4}$ & $1.82 \times 10^{5}$ & $-1.6 \times 10^{5}$ \\
\hline $10^{4}$ & 20 & $6.88 \times 10^{8}$ & $3.18 \times 10^{6}$ & $3.15 \times 10^{6}$ & $2.91 \times 10^{6}$ & $3.16 \times 10^{6}$ \\
\hline $10^{4}$ & 50 & $4.21 \times 10^{9}$ & $1.81 \times 10^{7}$ & $1.72 \times 10^{7}$ & $1.82 \times 10^{7}$ & $1.96 \times 10^{7}$ \\
\hline
\end{tabular}

${ }^{a}$ Reference 1. 

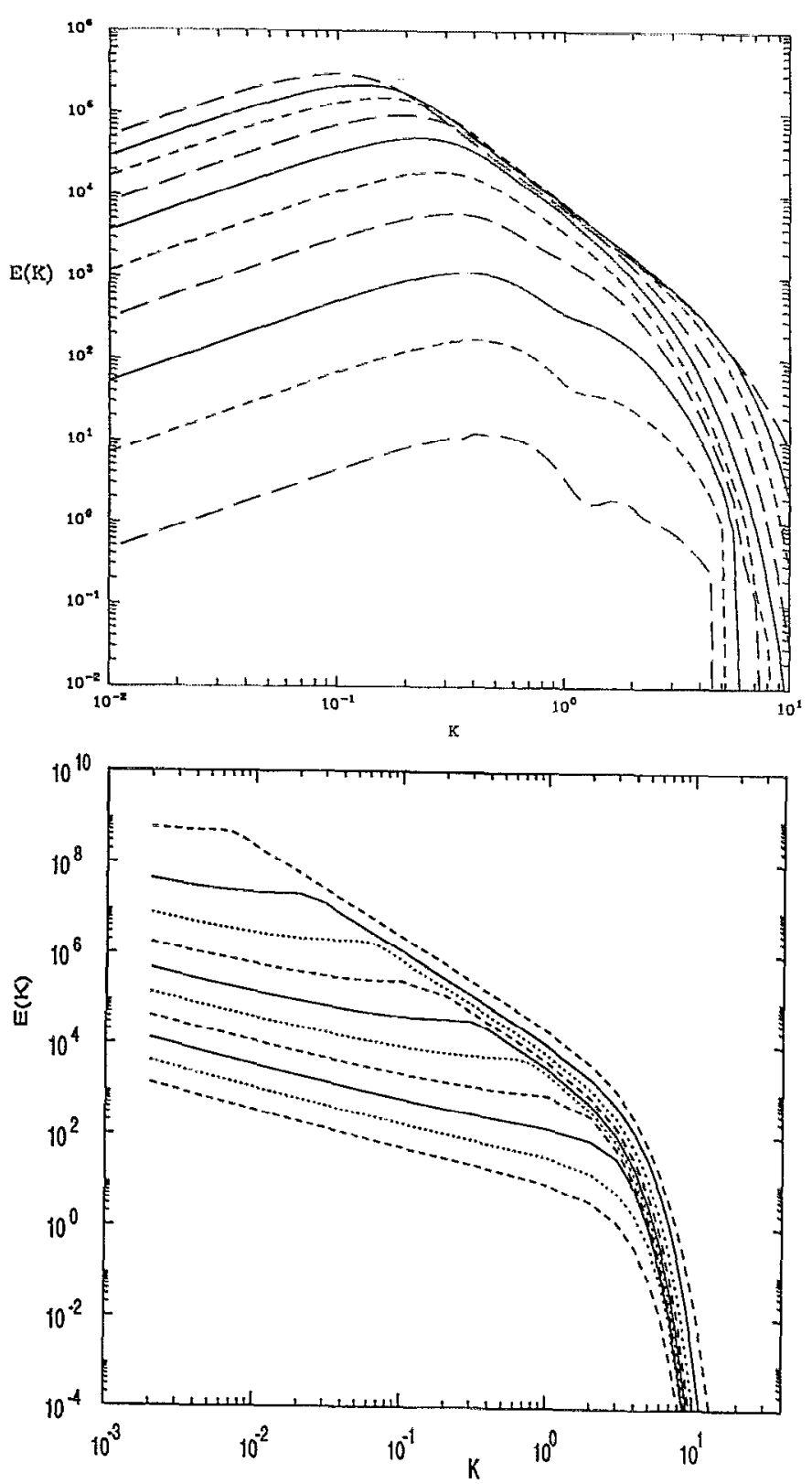

FIG. 5. Instantaneous nonaxisymmetric energy spectrum $\tilde{E}_{s}(K, a t), \alpha$ $=-0.5, \quad \Gamma_{0} / \nu=10^{3}, \quad\left(R^{2} a / 4 \nu\right)^{1 / 2}=20$. (a) Numerical double-integral method. (b) Stationary-phase numerical method. In both cascs times from upper curves downward are $a t=0.5,1.0,1.5,2.0,2.5,3.0,3.5,4.0,4.5,5.0$.

tions to the time integral near $a \tau=O(1)$. In Fig. 7 the spectra of Fig. 6 are plotted in the form $K^{5 / 3} E_{s}(K)$ on a loglinear plot. It is evident that the spectrum given by the stationary-phase-numerical method lies somewhere between $\exp \left(-B_{1} K^{2}\right)$ and $\exp \left(-B_{1} K\right)$ and is perhaps closer to the latter. The same may be true of the dissipation spectrum calculated in Sec. III but we have not verified this numerically.

From this section we may conclude that the stationaryphase evaluation of the integrals in the $L$ asymptotics is adequate but not excellent when compared to direct numerical evaluation of the integrals. The asymptotics of the time integration is good in the inertial range but poor in the dissipa-

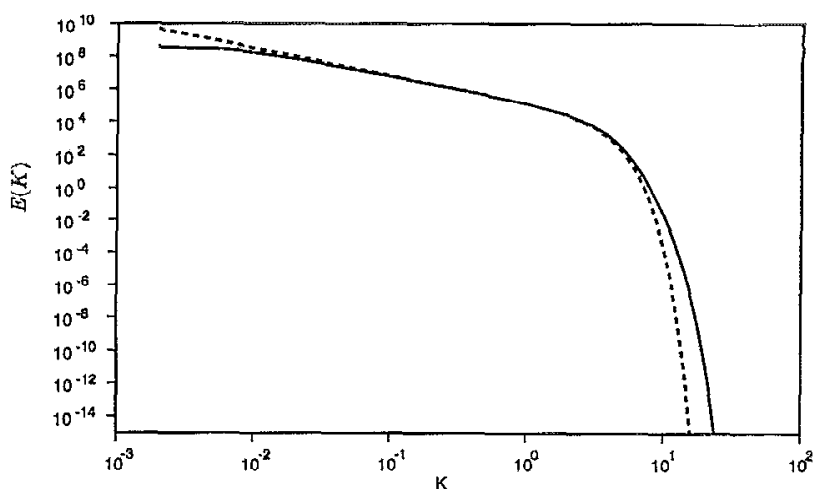

FIG. 6. Time-averaged nonaxisymmetric energy spectrum $E_{s}(K) . \alpha=-0.5$, $\Gamma_{0} / \nu=10^{3},\left(R^{2} a / 4 \nu\right)^{1 / 2}=20$. Lundgren $k^{-5 / 3}$ asymptotics.

tion range and this leads to a significant asymptotic under estimate of the dissipation range spectrum. For reasons that are not fully understood these errors in the inertial and dissipation range seem to approximately cancel in the calculation of the dissipation integral over the range of $\Gamma_{0} / \nu$ and $\left(a R^{2} / 4 \nu\right)^{1 / 2}$ surveyed, giving good agreement between numerics and the $L$ asymptotics.

\section{AN EXTENDED MODEL}

\section{A. An upper cutoff time and volume filling properties}

PS put the upper cutoff time $\tau_{2}=\infty$ in all calculations but found that this placed an upper bound on values of $\Gamma_{0} / \nu \sim O(100)$ above which the axisymmetric part of the spectrum was dominant. Although PS did not consider the space filling properties of the structures, it is clear that, if there exists a process which creates $N$ unit lengths of vortex per unit time per unit volume, then the vortex lifetime must be finite in order not to overfill space. The spiral lifetime is limited by the process of viscous diffusion between turns and is approximated by $\mathrm{PS}(25)$

$$
a \tau_{\mathrm{spir}}=C\left(\frac{R^{2} a}{4 \nu}\right)\left(\frac{\Gamma_{0}}{\nu}\right)^{-2 / 3},
$$

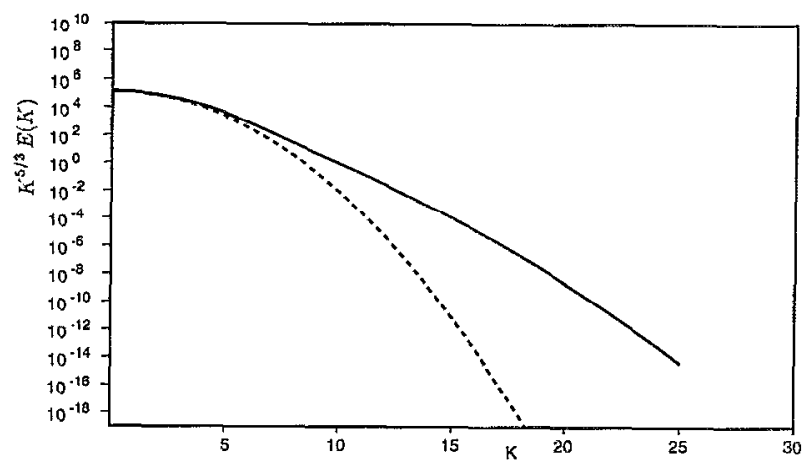

FIG. 7. Time-averaged nonaxisymmetric energy spectrum $E_{s}(K)$ on loglinear axes. $\alpha=-0.5, \Gamma_{\mathrm{n}} / \nu=10^{3},\left(R^{2} a / 4 \nu\right)^{1 / 2}=20$. , stationary-phase numerical method, .... Lundgren $k^{-5 / 3}$ asymptotics. 
TABLE II. (A) Calculated values of $K_{R}$ and $K_{p}$ and ratio of spectra, $\Gamma(\infty)=0$. Fourth column gives the maximum ratio of spectra components when $a \tau_{2}=\infty$, (B) Calculated values of $K_{R}$ and $K_{p}$ and ratio of spectra. $\Gamma(\infty) \neq 0$.

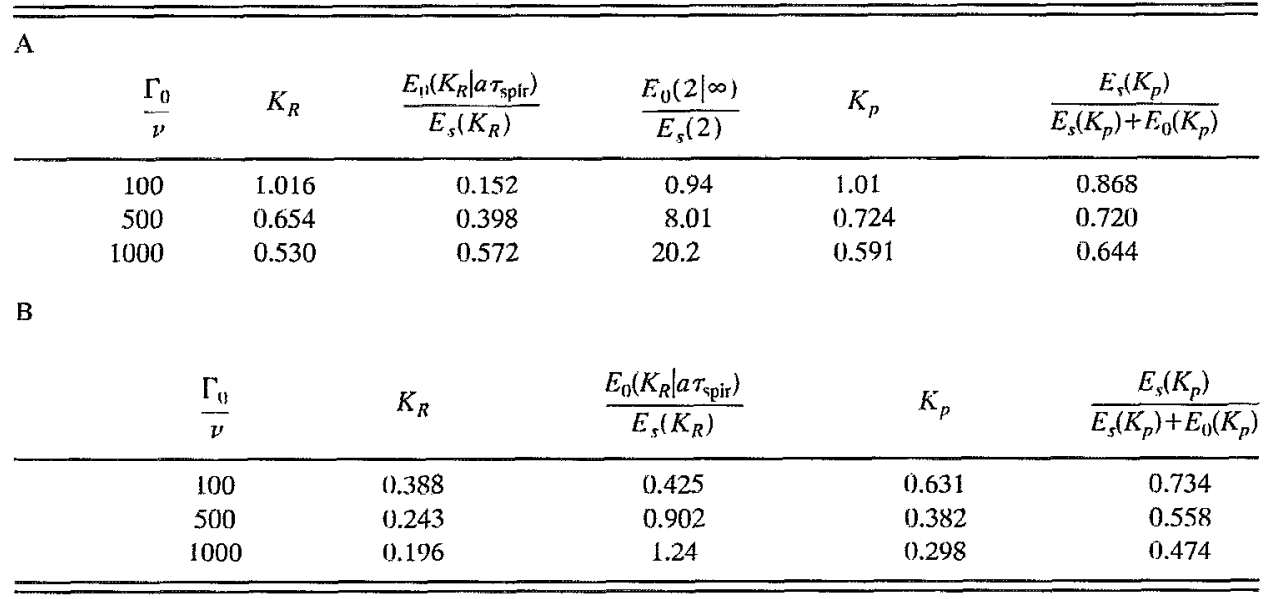

where $C$ is a constant which can be estimated from $\mathrm{PS}(2)$ and the present Eqs. (6) $-(8)$ and Eqs. (30)-(33) as $C \approx 15$. The present numerical simulations, however, all showed effective convergence of the integral (13) in a time given by Eq. (55) with $C \approx 10$, and in the estimates to follow we will adopt this value.

We now introduce two model assumptions. The first is that

$$
a \tau_{2}=a \tau_{\text {spir }},
$$

where the right-hand side is given by Eq. (55). Equation (56) supposes that there exists some mechanism for limiting the lifetime of the vortex core, such as vortex reconnection or vortex core instability. We justify Eq. (56) on the grounds that larger $\tau_{2}$ would give a core-dominated spectrum unless $\Gamma_{0} / \nu<O(100)$ while smaller $\tau_{2}$ would not allow the spiral roll-up process to proceed to completion, and there would be no inertial range. Therefore, Eq. (56) may be viewed as a consistency hypothesis when $\Gamma_{0} / \nu \gg O(100)$. Substitution of Eqs. (55) - (56) into Eq. (38) then gives the core spectrum at the cutoff, which we write in the form

$$
\begin{aligned}
E_{0}\left(K \mid a \tau_{\text {spir }}\right)= & N R^{2} \Gamma_{0}^{2} a^{-1 / 2} \nu^{-1 / 2} K \\
& \times \exp \left(-K^{2} / 2\right) A\left[\frac{K^{2}\left(\Gamma_{0} / \nu\right)^{2 / 3}}{4 C}\right], \\
A\left[\frac{K^{2}\left(\Gamma_{0} / \nu\right)^{2 / 3}}{4 C}\right]= & \frac{1}{32 \pi} \int_{K^{2}\left(\Gamma_{0} / \nu\right)^{2 / 3 / 4 C}}^{\infty} e^{-2 v}[v-1 \\
& \left.+M\left(-\frac{\alpha}{2}, 1, v\right)\right]^{2} \frac{1}{v^{2}} d v .
\end{aligned}
$$

The ratio $E_{0}\left(K \mid a \tau_{\text {spir }}\right) / E_{s}(K)$ is then, using Eq. (40)

$$
\begin{aligned}
\frac{E_{0}\left(K \mid a \tau_{\text {spir }}\right)}{E_{s}(K)}= & \frac{1}{D_{0}}\left(\frac{\Gamma_{0}}{\nu}\right)^{4 / 3} K^{8 / 3} \\
& \times \exp \left(-K^{2} / 3\right) A\left[\frac{K^{2}\left(\Gamma_{0} / \nu\right)^{2 / 3}}{4 C}\right] .
\end{aligned}
$$

The right-hand side of Eq. (59) has a maximum we denote by $K=K_{R}$ which is a function only of $\left(\Gamma_{0} / \nu\right)^{2 / 3} / 4 C$. This can be found numerically and calculated values are given in Table II A obtained with $C=10$. Also tabulated are the corresponding ratios $E_{0}\left(K_{R} \mid a \tau_{\text {spir }}\right) / E_{s}\left(K_{R}\right)$, the latter calculated using $\alpha=-0.5$ and $D_{0}=7.03$ (see Table VI). If in lieu of the cutoff (56) we put $a \tau_{2}=\infty$ as used by PS then the lower limit of integration in Eq. (58) may be set to zero which gives $A=B_{0}$ where $B_{0}(\alpha)$ is the parameter defined by $\operatorname{PS}(35)$. The right-hand side of Eq. (57) then has a maximum at $K_{R}=2$ with corresponding $E_{0}(K=2 \mid \infty) / E_{s}(K=2)$ given in the fourth column of Table II A, and calculated using $B_{0}(-0.5)=0.00848$ (PS Table I). Thus the cutoff (56) allows a much larger $\Gamma_{0} / \nu$ than that used by PS while retaining a $k^{-5 / 3}$ spectrum which is not buried by the $E_{0}$ component at large $K$. From Eqs. (57)-(58) may also be obtained the core dissipation at the cutoff $\epsilon\left(a \tau_{\text {spir }}\right)$ $=2 \nu \int_{0}^{\infty} k^{2} E_{0}\left(K \mid a \tau_{\text {spir }}\right) d k$. The double integral can be reduced to a single integral using exchange of the limits of integration in the $\nu-k$ plane. Setting $a=4, \nu=1, N=1$ as in Sec. IV we obtain, for $\Gamma_{0} / \nu=10^{3},\left(a R^{2} / 4 \nu\right)^{1 / 2}=5,20,50$, values $\epsilon\left(a \tau_{\text {spir }}\right)=4.5 \times 10^{3}, 6.6 \times 10^{4}$, and $4.5 \times 10^{5}$, respectively, which, from Table I may be seen to be small compared to the spiral dissipation.

Our second assumption is that the structures are approximately space filling. We suppose that a structure is created at time $t_{1}$, or equivalently $\tau_{1}=\phi / a$, and dies at time $a \tau_{2}$ given by Eq. (55). If the vortex is of lateral extent of $O(R)$ at $t_{1}$, then if it occupies the same volume in $t_{1}<t<t_{2}$ [this should be true up until the final viscous decay period, during which the radius will be constant and approximately equal to the Burgers radius $\left.2(\nu / a)^{1 / 2}\right]$, then the fraction of the total volume occupied by the vortices will be approximately

$$
\frac{V_{\text {vortex }}}{V}=2 \pi\left(\frac{N R^{2}}{a}\right)\left(a t_{2}-a t_{1}\right),
$$

The factor of 2 is included on the right-hand side of Eq. (60) first, to allow for the fact that the contribution to integral (41) determining $D_{0}(\alpha)$ comes from within a circle of radius ap- 
TABLE III. Calculated values of $N R^{2} / a, a^{2} \nu / \epsilon, . \pi_{0}, S_{3}, R_{\lambda}$ for $\alpha=-0.5$, $\phi=1$. Top; $\Gamma_{0} / \nu=500$. Bottom; $\Gamma_{0} / \nu=1000$.

\begin{tabular}{rrrrrr}
\hline \hline$\frac{a R^{2}}{4 \nu}$ & $\frac{N R^{2}}{a}$ & $\frac{a^{2} v}{\epsilon}$ & $\not T_{0}$ & \multicolumn{1}{c}{$S_{3}$} & \multicolumn{1}{c}{$R_{\lambda}$} \\
\hline 16 & 0.279 & 0.0129 & 2.14 & -0.357 & 45 \\
25 & 0.175 & 0.0198 & 1.75 & -0.430 & 215 \\
36 & 0.131 & 0.0258 & 1.60 & -0.478 & 732 \\
49 & 0.108 & 0.0310 & 1.48 & -0.512 & 2020 \\
64 & 0.093 & 0.0354 & 1.39 & -0.536 & 4814 \\
81 & 0.082 & 0.0393 & 1.33 & -0.555 & 10287 \\
100 & 0.075 & 0.0428 & 1.27 & -0.569 & 20194 \\
& & & & & \\
16 & 0.607 & 0.0039 & 3.32 & -0.204 & 10 \\
25 & 0.284 & 0.0080 & 2.51 & -0.287 & 54 \\
36 & 0.191 & 0.0116 & 2.17 & -0.341 & 195 \\
49 & 0.147 & 0.0149 & 1.97 & -0.381 & 556 \\
64 & 0.122 & 0.0179 & 1.84 & -0.411 & 1354 \\
81 & 0.105 & 0.0204 & 1.74 & -0.435 & 2940 \\
100 & 0.093 & 0.0228 & 1.66 & -0.455 & 5843 \\
\hline \hline
\end{tabular}

proximately $\sqrt{2} R$ (the effective vortex radius is larger that $R$ ), and second, because the tubes cannot perfectly pack the volume. We fix $\phi=1$ and put the left side of Eq. (60) equal to unity. This together with Eqs. (55) and (56) [and $\left.a t_{2}=\log \left(a \tau_{\text {spir }}+1\right)\right]$ then gives an equation containing the model parameters.

Equations PS(37), (38), and the present Eq. (60) (with the left side unity) are three equations for the five quantities $\Gamma_{0} / \nu, N R^{2} / a, \mathscr{K}_{0}, a^{2} \nu / \epsilon$, and $a R^{2} / 4 \nu$. In evaluating the core contribution to the dissipation we use the present Eqs. (57)-(58) in lieu of PS(34) to account for the finite cutoff. There is a weak dependence on $\phi$ since $a t_{1}=\log (1+\phi)$ in Eq. (60). We take $a R^{2} / 4 \nu$ as a free parameter, fix $\Gamma_{0} / \nu$ and $\phi=1.0$, and solve thesc equations for the rcmaining thrce parameters. When this is done the skewness $S_{3}$

$$
S_{3}=\frac{\overline{\overline{(\partial u / \partial x)^{3}}}}{\overline{\left(\overline{(\partial u / \partial x)^{2}}\right)^{3 / 2}}}
$$

can be computed from direct evaluation of the average value of $(\partial u / \partial x)^{3}$. Using the model, this leads to the formula

$$
S_{3}=\frac{2}{35}(15)^{3 / 2}\left[4\left(\frac{a^{2} \nu}{\epsilon}\right)^{3 / 2}-\left(\frac{a^{2} \nu}{\epsilon}\right)^{1 / 2}\right]
$$

This equation is derived as Eq. (C8) in Appendix C.

Table III shows calculated results for $\Gamma_{0} / \nu=500,1000$ tabulated against $a R^{2} / 4 \nu$. Also shown are values of the Taylor Reynolds number $R_{\lambda}$, the calculation of which, in terms of the model parameters, is discussed in the next section. By comparison PS use $\Gamma_{0} / \nu=100$ and find $\mathscr{H}_{0}=1.55$, $S_{3}=-0.34$, and $a^{2} \nu / \epsilon=0.0126$ independent of $a R^{2} / 4 \nu$. From Table III it may be seen that the present model gives a decrease in $\mathscr{C}_{0}$ and a slow increase in $S_{3}$ with increasing $a R^{2} / 4 \nu$ and $R_{\lambda}$, the latter trend being in qualitative agreement with the experimental compilation of Van Atta and Antonia. ${ }^{13}$ As an alternative to Eq. (62) for calculating the skewness, we may use Kármán-Howarth equation; see PS(40). It is shown in Appendix C that when $a \tau_{2}=\infty$, the two approaches give exactly the same answer, which is a check on the dynamic self-consistency of the model. With the present finite cutoff $\tau_{2}$, the unsteady terms in the Kárman-Howarth equation must be included and the necessary corrections are derived in Appendix C. The two methods were found to give eight to ten figure agreement for $S_{3}$ over the range of parameters given in Table III which is a further check on dynamical consistency.

\section{B. The Taylor Reynolds number}

We seek an alternative closure to Eq. (1) which will relate the the model parameters to the Taylor Reynolds number $R_{\lambda}$. Since the problem of relating the small-scale vortex structures to the energy containing eddies is unsolved, we appeal to scaling arguments, and note that, in what follows, several dimensionless quantities which appear in scaling relations are assigned arbitrary values of $O(1)$. We denote the initial longitudinal scale of the vortex structure (i.e., in the direction of the stretching strain) by $2 L_{1}$. At the upper cutoff $t_{2}$ the stretched length is

$$
2 L_{2}=2 L_{1} e^{a t_{2} \approx 2 L_{1} a \tau_{2}=2 L_{1} a \tau_{\text {spir }} .}
$$

The velocity near the end of the structure due to the strain field is $a L_{2}$. We now assume that this velocity can be equated to the typical velocity of a large scale

$$
u=a L_{2} \text {, }
$$

where $u$ is the large-scale velocity $u=\left(\overline{u_{1}^{2}}\right)^{1 / 2}$ and the turbulent kinetic energy is $3 \overline{u_{1}^{2}} / 2$. Equation (64) says that, on the average, the large scale strain $a$ is coherent over a length with upper bound $L_{2}$. From a physical standpoint (64) seems natural since if there existed stretched vortex lengths exceeding $L_{2}$, continuous stretching would generate, on average, velocities larger than $u$, which produces an inconsistency. We cannot, however, rule out $a L_{2}<u$.

Use of the relations for isotropic turbulence

$$
R_{\lambda}=\frac{u \lambda}{\nu}, \quad \epsilon=\frac{15 \nu u^{2}}{\lambda^{2}},
$$

where $\lambda$ is the Taylor microscale, and Eq. (55) with Eq. (56) is then sufficient to obtain

$$
R_{\lambda}=4 \sqrt{15} C^{2}\left(\frac{L_{1}}{R}\right)^{2} \frac{\left(a R^{2} / 4 \nu\right)^{3}}{\left(\Gamma_{0} / \nu\right)^{4 / 3}}\left(\frac{a^{2} \nu}{\epsilon}\right)^{1 / 2} .
$$

We now put

$$
\tilde{A}=\frac{\epsilon L}{u^{3}},
$$

where $L$ is the integral scale. We will subsequently take $\tilde{A}=$ const $=1$. Sreenivasan ${ }^{14}$ collates experimental evidence supporting $\tilde{A}=1$ for square-mesh grids for $R_{\lambda}>50$ but finds larger values for other types of grid. From Eqs. (64)-(67) may be obtained

$\frac{L}{L_{2}}=\frac{\pi}{\sqrt{15}}\left(\frac{a^{2} \nu}{\epsilon}\right)^{1 / 2} R_{\lambda}, \frac{\lambda}{L_{2}}=\sqrt{15}\left(\frac{a^{2} \nu}{\epsilon}\right)^{1 / 2}$.

Equation (66) still contains $L_{1} / R$. We now assume $2 L_{1}=R$, i.e., the longitudinal initial scale is of order the 
TABLE IV. Calculated values of length scale ratios. $\alpha=-0.5, \Gamma_{0} / \nu=10^{3}$, $\phi=1 . A=1$.

\begin{tabular}{crrrrr}
\hline \hline$\frac{a R^{2}}{4 \nu}$ & \multicolumn{1}{l}{ L } & $\frac{\lambda}{L_{2}}$ & $\frac{L}{R}$ & $\frac{\lambda}{R}$ & $R_{\lambda}$ \\
\hline 23.8 & 1 & 0.34 & 1.2 & 0.4 & 45 \\
36 & 5 & 0.42 & 9.8 & 0.75 & 195 \\
64 & 47 & 0.52 & 149 & 1.6 & 1354 \\
100 & 228 & 0.58 & 1138 & 2.9 & 5843 \\
\hline
\end{tabular}

lateral initial scale. Use of this in Eq. (66) then gives a relation between $R_{\lambda}$ and the model parameters. Typical values are listed in Tables III and IV. Thus, for fixed $\Gamma_{0} / \nu$ the results of Sec. V A allow all dimensionless groups to be calculated in terms of $R_{\lambda}$. It now may also be shown that

$$
\begin{aligned}
& \frac{L}{R}=\frac{\tilde{A} C^{1 / 3}}{2 \times 15^{2 / 3}}\left(\frac{a^{2} \nu}{\epsilon}\right)^{1 / 3}\left(\frac{\Gamma_{0}}{\nu}\right)^{-2 / 9} R_{\lambda}^{4 / 3}, \\
& \frac{\lambda}{R}=\frac{15^{1 / 3} C^{1 / 3}}{2}\left(\frac{a^{2} \nu}{\epsilon}\right)^{1 / 3}\left(\frac{\Gamma_{0}}{\nu}\right)^{-2 / 9} R_{\lambda}^{1 / 3} .
\end{aligned}
$$

Table III shows that $a^{2} \nu / \epsilon$ is an increasing function of $R_{\lambda}$. Therefore, from the first of Eq. (68) $L \Rightarrow L_{2}$ at large $R_{\lambda}$. This is illustrated in Table IV where all length scales are tabulated against $R_{\lambda}$. When $R_{\lambda}=45$ we find $L / L_{2}=1$, which suggests that this value may be interpreted as a Reynolds number below which there is no turbulence. We remark that $\lambda / L_{2}=O(1)$ while $\lambda / R$ increases slowly with $R_{\lambda}$ and remains $O(1)$ at $R_{\lambda}=O(1000)$. Since the vortex structures can only provide an inertial range down to $k R=O(1)$ (which in the stretched vortex is provided by the tightening of the spiral turns) then at very large $R_{\lambda}$ the full inertial range would require either some other mechanism or a distribution of vortex scales (i.e., a distribution of $R$ ) for which we might interpret the present fixed scalings (with $R_{\lambda}$ ) as averages.

Jimenez ${ }^{3}$ et al. study the structure of the intense vorticity in numerical simulations of isotropic turbulence at $R_{\lambda} \approx 170$. They find that the most intense vorticity, defined as vorticity with magnitude greater than several times the root mean square vorticity $\omega^{\prime}$, is organized into tubes or ribbons with length of order the integral scale $L$. However these intense regions appear to make negligible contribution to the enstrophy, and occupy negligible volume. They also find that the so-called background vorticity, with magnitude between $\omega^{\prime}$ and that of the intense regions is also organized into coherent structures that account for some $80 \%$ of the enstrophy. Visual inspection of the the right-hand part of their Fig. 5, which shows the background vorticity at $R_{\lambda}=170$, appears to indicate the presence of structures with lengths of order $L / 5$. Our Table IV shows that at $R_{\lambda}=196$, the maximum lengths of the spiral structures $\left(2 L_{2}\right)$ is about $L / 3$. These estimates are not inconsistent if it assumed that the structures observed by Jiméne $z^{3}$ et al. are still undergoing stretching at the instant of the snapshot, and thus may not have reached their full lengths. Thus, although these background structures may not contain vorticity field phase-amplitude correlations with

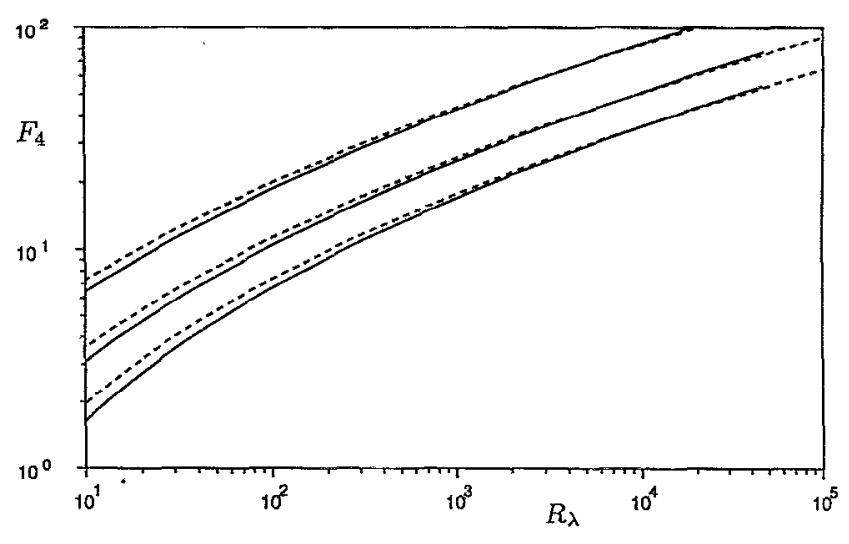

FIG. 8. Flatness factor $F_{4}$ vs $R_{\lambda} . \cdots, \Gamma_{\nu} / \nu=500 .-, \Gamma_{\nu} / \nu=1000$. Top; $\phi=0.75$. Middle; $\phi=1.00$. bottom; $\phi=1.25$.

the strong coherence of the present model, they may nevertheless provide candidates for fine-scale vortices of the Lundgren-Townsend type.

\section{Velocity derivative moments}

PS calculated velocity derivative and vorticity moments from an analysis of the strain field interior to a vortex coupled to an integration over the vortex cross section and lifetime, and an average over the assumed random orientation of the vortex structures in space. Their Eq. (84a) gives the $2 p$ th moment of the vorticity in the structure as

$$
\begin{gathered}
\overline{\omega^{2 p}}=2^{2 p-3} a^{2 p} \hat{L}_{2 p}(\phi) \hat{K}_{2 p}\left(\frac{N R^{2}}{a}\right)\left(\frac{\Gamma_{0}}{\nu}\right)\left(\frac{a^{2} R}{4 \nu}\right)^{p-3 / 2}, \\
p \geqslant 2,
\end{gathered}
$$

where the quantities $\hat{L}_{2 p}(\phi), \hat{K}_{2 p}$ are calculated from the detailed vorticity distribution. The moments of the longitudinal velocity-derivative field are then

$$
\begin{aligned}
& \overline{\left(\frac{\partial u}{\partial x}\right)^{2 p}}=\hat{U}_{2 p} \overline{\omega^{2 p}}, \\
& \overline{\left(\frac{\partial u}{\partial x}\right)^{2 p+1}}=-a \hat{V}_{2 p} \overline{\omega^{2 p}},
\end{aligned}
$$

where the numbers $\hat{U}_{2 p}$ and $\hat{V}_{2 p}$ follow from integration over the orientations of the structures [the definition of the overbar and double-overbar notation is given by PS(50)]. The hyperflatness and hyperskewness factors

$$
\begin{gathered}
F_{2 p} \equiv \overline{(\partial u / \partial x)^{2 p} / \overline{(\partial u / \partial x)^{2}}}, \\
S_{2 p+1} \equiv(\partial u / \partial x)^{2 p+1} / \overline{(\partial u / \partial x)^{2}}
\end{gathered}
$$

can then be calculated. We presently repeat this process replacing the closure (1) with the present Eq. (66) together with the present calculated dependence on $a R^{2} / 4 \nu$ of the groups in Eq. (71) and in the expression for $\overline{(\partial u / \partial x)^{2}}$. The results for $F_{4}$ obtained with $\Gamma_{0} / \nu=500,1000$ are plotted vs $R_{\lambda}$ in Fig. 8. The results are rather insensitive to $\Gamma_{0} / \nu$ but show dependence on the early cutoff time defined by $\phi$. They 
TABLE V. Comparison of velocity derivative moments

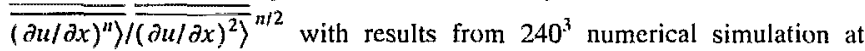
$R_{\lambda}=150$ (Ref. 6). $A ; \alpha=-0.5, \Gamma_{0} / \nu=500, \phi=1.0 . B ; \alpha=-0.5, \Gamma_{0} / \nu=1000$, $\phi=1.0$

\begin{tabular}{|c|c|c|c|c|}
\hline \multirow[b]{2}{*}{$n$} & \multirow{2}{*}{$\begin{array}{l}\text { V\&M } \\
\text { No tails }\end{array}$} & \multirow{2}{*}{$\begin{array}{c}\text { V\&M } \\
\text { Exp. Tails }\end{array}$} & \multicolumn{2}{|c|}{ Present } \\
\hline & & & A & $B$ \\
\hline 4 & 5.85 & 6.00 & 13.0 & 12.0 \\
\hline 5 & $-1.01 \times 10^{1}$ & -9.92 & -12.4 & -8.85 \\
\hline 6 & $1.05 \times 10^{2}$ & $1.32 \times 10^{2}$ & $3.36 \times 10^{2}$ & $2.75 \times 10^{2}$ \\
\hline 7 & $-3.89 \times 10^{2}$ & $-3.39 \times 10^{2}$ & $-4.92 \times 10^{2}$ & $-3.11 \times 10^{2}$ \\
\hline 8 & $4.33 \times 10^{3}$ & $9.36 \times 10^{3}$ & $1.72 \times 10^{4}$ & $1.24 \times 10^{4}$ \\
\hline 9 & $-2.69 \times 10^{4}$ & $-1.67 \times 10^{4}$ & $-3.40 \times 10^{4}$ & $-1.9 \times 10^{4}$ \\
\hline 10 & $3.45 \times 10^{5}$ & $1.34 \times 10^{6}$ & $1.1 \times 10^{6}$ & $7.4 \times 10^{5}$ \\
\hline 11 & $-3.11 \times 10^{6}$ & $-8.49 \times 10^{5}$ & $-2.9 \times 10^{6}$ & $-1.4 \times 10^{6}$ \\
\hline 12 & $4.61 \times 10^{7}$ & $2.58 \times 10^{8}$ & $8.9 \times 10^{7}$ & $5.1 \times 10^{7}$ \\
\hline
\end{tabular}

show a rather steeper increase with $R_{\lambda}$ than the $R_{\lambda}^{1 / 4}$ powerlaw found by PS. The higher order moments are compared in Table $\mathrm{V}$ with unpublished results of Vincent and Meneguzzi ${ }^{6}$ calculated from $240^{3}$ numerical simulations of isotropic turbulence at $R_{\lambda} \approx 150$.

\section{CONCLUDING REMARKS}

We have demonstrated that a model of turbulent flow consisting of stretched spiral vortices (originally suggested by Lundgren ${ }^{1}$ ) can be used to make predictions about properties of homogencous turbulence. For example, the dependence of the Kolmogorov constant and skewness coefficient on Reynolds number can be estimated, and energy dissipation and squared vorticity spectra can be obtained. Also, the model suggests that the energy and enstrophy spectra are composed of two components arising from the asymmetric spiral and axisymmetric core part of the spiral vortex, which should produce at large Reynolds number an observable bump in the vicinity of the peak of the enstrophy spectrum at a wave number $k_{p}$, say. A recent examination of experimental spectra by She and Jackson ${ }^{15}$ shows this feature. (It is also seen in some numerical simulations.) These authors also show that the energy and vorticity spectra collapse when scaled with $E\left(k_{p}\right)$, while the spectra scaled against $\left(\epsilon \nu^{5}\right)^{1 / 4}$ and wave numbers against $\left(\epsilon / \nu^{3}\right)^{1 / 4}$ depend upon Reynolds number. We now show that the model has this property provided, as has been assumed, that the vortex Reynolds number $\Gamma_{0} / \nu$ and other model parameters such as $C$ and $\alpha$ are independent of $R_{\lambda}$.

For we have

$$
\begin{aligned}
E(K)= & E_{0}(K)+E_{s}(K)=\frac{N R^{2}}{a}\left(\frac{\Gamma_{0}}{\nu}\right)^{2 / 3}\left(\frac{\nu a^{2}}{\epsilon}\right)^{1 / 4}\left(\epsilon \nu^{5}\right)^{1 / 4} \\
& \times\left[A\left(\frac{\Gamma_{0}}{\nu}\right)^{4 / 3} K e^{-K^{2} / 2}+D_{0} K^{-5 / 3} e^{-K^{2} / 6}\right],
\end{aligned}
$$

where $A$ is defined by Eq. (58) and the reader is reminded that $K=2 k(v / a)^{1 / 2}$. Defining $K_{p}$ as the root of the equation

$$
\frac{d}{d K}\left(K^{2}\left(E_{0}(K)+E_{s}(K)\right)\right)=0,
$$

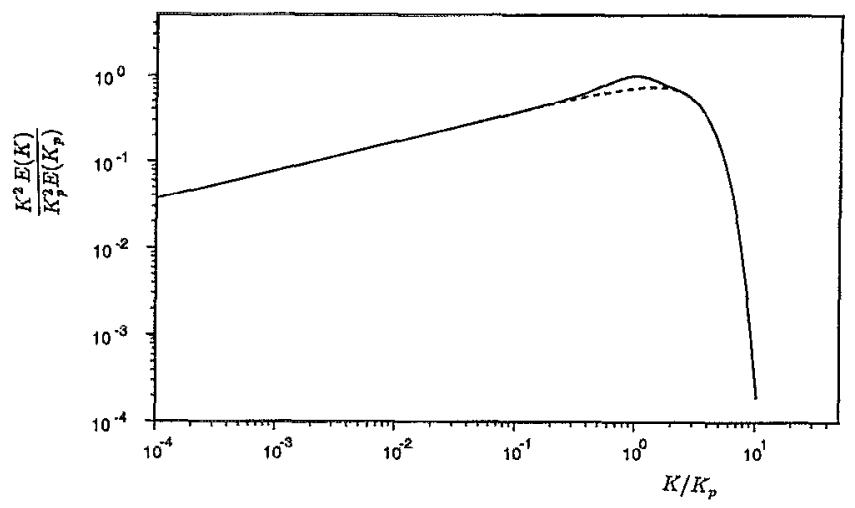

FIG. 9. Vorticity spectrum vs $K . \alpha=-0.5, \Gamma_{0} / \nu=500, \Gamma(\infty)=0$, finite upper cutoff $\tau_{2},-, K^{2} E(K) /\left(K_{p}^{2} E\left(K_{p}\right)\right), \cdots, K^{2} E_{s}(K) /\left(K_{p}^{2} E\left(K_{p}\right)\right)$.

we see that according to the model $K_{p}$ is independent of $R_{\lambda}$ because $A$ and $D_{0}$ are independent of $R_{\lambda}$, and hence $K^{2} E(K) / K_{p}^{2} E\left(K_{p}\right)$ depends only on $K$ and therefore only on $K / K_{p}=k / k_{p}$. We solve Eq. (75) for $K_{p}$ numerically with $C=10$ and $\Gamma_{0} / \nu$ fixed. Values for $\Gamma_{0} / \nu=100,500$, and 1000 are given in Table II $\mathrm{A}$ along with the corresponding ratio $E_{s}\left(K_{p}\right) / E\left(K_{p}\right)$. Figure 9 shows the bump in a plot of $K^{2} E(K) / K_{p}^{2} E\left(K_{p}\right)$. Also shown is $K^{2} E_{s}(K) / K_{p}^{2} E\left(K_{p}\right)$ vs $K / K_{p}$. The curves of Fig. 9 are not inconsistent with the data depicted in Fig. 2 of Ref. 15. The dimensional wave number $k_{p}$ is $2 K_{p}(a / \nu)^{1 / 2}$ and hence the dimensionless Kolmogorov scaled wave number

$$
k_{p}\left(\nu^{3} / \epsilon\right)^{1 / 4} \cong \frac{k_{p}}{k_{\eta}}=\frac{1}{2} K_{p}\left(a^{2} \nu / \epsilon\right)^{1 / 4}
$$

is, according to Table III, a slowly increasing function of $R_{\lambda}$. This is shown in Fig. 10 which compares Eq. (76) using values $a^{2} \nu / \epsilon$ obtained from Table III with data from Fig. 3 of Ref. 15.

There are, however, two conflicts. First, the analytical estimates of both $E_{0}$ and $E_{s}$ used presently have a Gaussian decay with large wave number rather than the observed exponential decay. At present, we do not regard this as a serious difficulty, since as was demonstrated in Sec. IV C, the asymptotics appears to underestimate the true model $E_{s}$ in

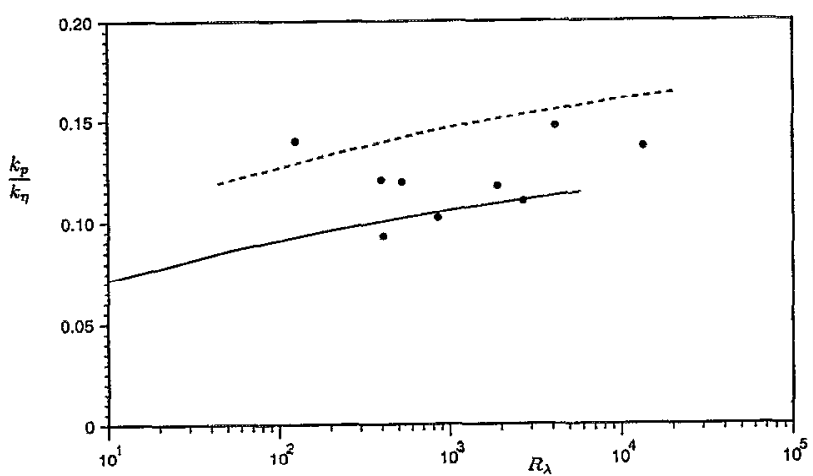

FIG. 10. $k_{p}\left(\nu^{3} / \epsilon\right)^{1 / 4}=k_{p} / k_{\eta}$ vs $R_{\lambda}, \cdots--, \Gamma_{0} / \nu=500 \ldots, \Gamma_{0} / \nu=1000, \Gamma(\infty)=0$, finite upper cutoff $\tau_{2}$. Data scaled from Fig. 3 of Ref. 15. 
TABLE VI. Values of $D_{0}, \Gamma(\infty)=0$ and $\Gamma(\infty) \neq 0$ for three values of $\alpha$.

\begin{tabular}{ccc}
\hline & $D_{\mathrm{n}}$ & $D_{0}$ \\
$\alpha$ & $\Gamma(\infty)=0$ & $\Gamma(\infty) \neq 0$ \\
\hline-0.25 & 12.19 & 15.85 \\
-0.5 & 7.03 & 8.31 \\
-1.0 & 2.21 & 2.41 \\
\hline
\end{tabular}

the dissipation range while, surprisingly, providing good approximations to the integrals determining $\epsilon$. Alternatively PS showed that adding noise to the values of the parameters converts the Gaussian to an exponential decay. In other words, this is a consequence of the clearly unsatisfactory assumption that the parameters have the same values for all structures.

Second, our results predict that the modification to the Kolmogorov spectrum is proportional to $K$, not $K^{-1}$. Figure 9 compared with Fig. 2 of Ref. 15 shows, however, that this difference is hard to detect in the neighborhood of the dissipation cutoff. The $K$ dependence is due to the assumption [see Eq. (34)] that $\Gamma(\infty)=0$, i.e., the total strength of the spiral structure is zero. This assumption is attractive as it ensures that the mean vorticity is zero, whatever the orientation of the structures, and also gives rise to a finite life time for the structures, so that a long-time cutoff is not necessary, although as discussed in Sec. II it is desirable. This choice also allows vortex lines to close at the end of the structures and avoids the problem of open vortex lines which occurs with $\Gamma(\infty) \neq 0$. However, it may not be appropriate and alternatives should be considered.

With a long-time cutoff $\tau_{2}$, it is possible to investigate the properties of a model with $\Gamma(\infty) \neq 0$. The simplest way to proceed is to follow the analysis of Sec. II D and put $g=0$ in Eq. (29). Eqs. (40)-(41) remain unaltered, and the asymmetric part of the spectrum is still given by Eqs. (40)-(41) except for one quantitative difference in that the function $\bar{\Lambda}(\xi)$ in the denominator of (41) now involves only $\bar{f}$ [see Eqs. (7)-(8)]. The new values of $D_{0}$ (Table VI) are slightly higher.

In contrast, there are qualitalive changes to the contribution to the spectrum from the axisymmetric core. The formula for $E_{0}$ is again Eq. (37), but the integrand in the integral for $A$ is altered. Now,

$$
A=\frac{1}{32 \pi} \int_{u_{2}}^{u_{1}} e^{-2 u}\left[M\left(-\frac{\alpha}{2}, 1, u\right)\right]^{2} \frac{d u}{u^{2}},
$$

As before, we take $u_{1}=\infty$ and $u_{2}=K^{2}\left(\Gamma_{0} / \nu\right)^{2 / 3} / 4 C$.

When $\Gamma_{0} / \nu$ is large, $A \sim(C / 8 \pi)\left(\Gamma_{0} / \nu\right)^{-2 / 3} K^{-2}$ and

$$
E_{0} \sim \frac{C}{8 \pi} \frac{N R^{2}}{a}\left(\frac{\Gamma_{0}}{\nu}\right)^{4 / 3} \frac{1}{K} e^{-K^{2} / 2} .
$$

Notice the $K^{-1}$ behavior, suggested by She and Jackson ${ }^{15}$ from analysis of experimental data and by others on theoretical grounds (e.g., Yakhot and Zakharov ${ }^{16}$ ). Of course, it is obvious that the model with finite strength has the property that the structures will asymptote to Burgers vortices and hence the $K^{-1}$ behavior follows from Townsend's ${ }^{9}$ results.
With the cutoff given by Eq. (55) calculated values of $K_{R}$ and $K_{p}$ for this case, together with the corresponding maxima in the spectral ratios, are shown in Table II B.

Finally, we again emphasize the restrictions on the present results. For much of the present paper, we have considered a special realization of the Lundgren spiral vortex, consisting of a thin shear layer in the process of roll-up, whilst undergoing stretching in the direction of vortex lines. This is motivated by the idea of a formation process based on the roll-up of vortex sheets and has the attraction that the relative simplicity of the vorticity field allows progress in the analysis. The typical vorticity signature of such a structure would consist of something like a central "vortex tube" surrounded by "sheetlike" layers. In the latter stages of the evolution process, only the nearly axisymmetric central ember would remain, and this would be essentially a Burgers vortex if the total circulation were nonzero. A formation process different from the roll-up of vortex sheets can be expected to produce a dependencey of the $f_{n}$ on $n$, and therefore a vorticity field that may not resemble rolled-up shear layers; for example, if $f_{n} \sim n^{-1}$, a vorticity field more like the winding of a vorticity discontinuity than a velocity discontinuity, could be expected, with no sheetlike concentration of the vorticity field. Although the $k^{-5 / 3}$ form of the energy (velocity) spectrum is robust in the sense that it does not depend on the details of the $f_{n}$, provided the series in the first term of Eq. (29) converges, our results for the dissipation and vorticity-squared spectra indicate that this may not be true of the spectra of higher-order quantities. To date we know of no systematic search of experimental or numerical data bases for vortices which might resemble the stretchedspiral type, although we have identified some possible candidates in the work of Jimenez ${ }^{3}$ et al. The need to consider more general types of structure than the shear-layer prototype considered here, would need to be recognized in such an investigation.

\section{ACKNOWLEDGMENTS}

The authors wish to thank Professor D. W. Moore and Professor A. Leonard for helptul discussions. D. I. P. was partially supported by NSF Grant No. CTS-9311811 and P. G. S. was partially supported by the Department of Energy under Grant No. DE-FG03-89ER25073. We wish to thank Dr. Maurice Meneguzzi for providing unpublished data on velocity-derivative moments.

\section{APPENDIX A: THE ENERGY SPECTRUM}

Here we outline an alternative derivation for the leading order terms in the energy spectrum utilizing a form of the vorticity distribution in the Lundgren spiral given in PS, Appendix A. This is of interest since it does not require stationary phase evaluation of spectral integrals, and can therefore be used as an independent check on this method. In local coordinates $(x-y)$ in a region surrounding a point on the rolling-up thin shear-layer with extent much smaller than the local sheet radius of curvature $\rho$, and with $x$ tangential and $y$ normal to the layer which stretches to $\pm \infty$ in the $x$ direction, this takes the form 


$$
\hat{\omega}(y, \tau)=\frac{2 \pi f(\rho)}{\Lambda(\rho) \tau\left[\frac{4}{3} \pi \nu \tau\right]^{1 / 2}} \exp \left(-\frac{3 y^{2}}{4 \nu \tau}\right),
$$

where $y \sim[\theta-\Omega(\rho) \tau] / \Lambda(\rho) \tau$ is normal to the layer centerline. This approximation describes an unstretched or purely two-dimensional spiral evolution including the effect of the coarse-grained axisymmetric strain field $e_{\rho \theta}=\rho \Lambda(\rho) / 2$ felt locally by the thin shear layer. An analysis of the $\omega^{2 p}$ moments based on Eq. (A1) is given in Appendix B.

Equations (10)-(14) may be written in the equivalent form

$$
\begin{aligned}
E(k)= & \frac{N}{8 \pi^{2} k} \int_{\tau_{1}}^{\tau_{2}} d \tau \int_{0}^{2 \pi} d \theta_{k} \mid \int_{0}^{\infty} \int_{0}^{2 \pi} \exp \left[\frac{-i \rho k}{\sqrt{1+a \tau}}\right. \\
& \left.\times \cos \left(\theta-\theta_{k}\right)\right]\left.\hat{\omega}(\rho, \theta, \tau) \rho d \rho d \theta\right|^{2},
\end{aligned}
$$

where $\tau_{1}$ and $\tau_{2}$ are early and late cutoff times, respectively. We first consider $a \tau<1$ and make the approximation $\sqrt{1+a \tau} \sim 1$ in the exponential of Eq. (A2). Further, consider a length $2 D$ of the shear layer in $-D<x<D$ whose thickness of order $\sqrt{\nu \tau}$ is small compared to its radius of curvature. The contribution to the area integral in vertical braces in (7) from this section is $\int_{-D}^{D} \int_{-\infty}^{\infty} \cdots d x d y$ where locally $x=\rho \cos \theta, y=\rho \sin \theta$ and

$\exp \left[i k \rho \cos \left(\theta-\theta_{k}\right)\right]$

$$
=\exp \left[i k x \cos \theta_{k}+i k y \sin \theta_{k}\right] \text {. }
$$

Using Eq. (Al) the $x$ and $y$ integrals can now be evaluated to give

$$
\begin{aligned}
& \int_{0}^{2 \pi} \mid \int_{-D}^{D} \int_{-\infty}^{\infty} \exp \left[i k x \cos \theta_{k}+i k y \sin \theta_{k}\right] \\
& \quad \times\left.\hat{\omega}(y, \tau) d y d x\right|^{2} d \theta_{k} \\
& =\frac{32 \pi^{2} f^{2}(\rho)}{\Lambda^{2}(\rho) \tau^{2} k^{2}} \int_{0}^{\pi} \frac{\sin ^{2}\left(k D \cos \theta_{k}\right)}{\cos ^{2} \theta_{k}} \\
& \quad \times \exp \left(\frac{-2 k^{2} \nu \tau \sin ^{2} \theta_{k}}{3}\right) d \theta_{k} .
\end{aligned}
$$

On applying the transformation $\xi=\cos \theta_{k}$, the contribution per unit length of shear layer to the integral may be expressed as

$$
\begin{aligned}
\frac{1}{2 D} \int_{0}^{2 \pi}|\cdots|^{2} d \theta_{k}:= & \frac{16 \pi^{2} f^{2}(\rho)}{\Lambda^{2}(\rho) \tau^{2} k} \exp \left(\frac{-2 k^{2} \nu \tau}{3}\right) \\
& \times \int_{-1}^{1} \frac{\sin ^{2}(k D \xi)}{k D \xi^{2} \sqrt{1-\xi^{2}}} \exp \left(\frac{2 k^{2} \nu \tau \xi^{2}}{3}\right) d \xi
\end{aligned}
$$

Next we restrict attention to the range of wave numbers

$$
\frac{1}{D} \ll k \ll \sqrt{a / \nu} \text {. }
$$

The exponential under the integral in Eq. (A5) is then nearly unity and the integral can be easily calculated to be $\pi+O\left[(k D)^{-1}\right]$. We now consider a length $d s$ of the nearly circular spiral layer, which from Eq. (9) we can approximate $d s \approx \rho \Lambda(\rho) \tau d \rho$. Integrating in $0<\rho<\infty$ and substituting into Eq. (A2) then gives a contribution to $E_{s}(k)$ which we denote by $E_{s_{1}}$

$$
\begin{aligned}
E_{s_{1}}(k)= & \frac{2 \pi N}{k^{2}} \int_{\phi / a}^{\infty} \tau^{-1} \exp \left(\frac{-2 k^{2} \nu \tau}{3}\right) \\
& \times d \tau \int_{0}^{\infty} \frac{|f(\rho)|^{2} \rho d \rho}{|\Lambda(\rho)|},
\end{aligned}
$$

In Eq. (A7) the lower limit of integration is $\tau_{1}=\phi / a$ and we have taken $\tau_{2} \rightarrow \infty$ since the main contribution comes from small $a \tau$ and the $\tau$ integral is convergent at $\infty$. Putting $u=a \tau$, Eq. (A7) may be written as

$$
E_{s_{1}}(k)=\frac{2 \pi N}{k^{2}} E_{1}\left[\frac{2 \phi k^{2} \nu}{3 a}\right] \int_{0}^{\infty} \frac{|f(\rho)|^{2} \rho d \rho}{|\Lambda(\rho)|} .
$$

When $\phi \rightarrow 0, E_{s_{1}}$ is weakly divergent as $k^{-2} \log \phi$. Further, since Eq. (A8) has been obtained by setting $a=0$ in Eq. (A2) [note that the appearance of $a$ in Eq. (A8) is parametric through the definition of $\tau_{1}$ ], then it must be the asymptotic spectrum of the unstrained spiral.

An estimate for the $k^{-5 / 3}$ spectrum can also be obtained from $\mathrm{Eq}$. (A2) using the analysis leading to Eq. (A5). In obtaining Eq. (A5), we began by putting $a=0$ in Eq. (7). We now repeat the integrations but replace $k$ by $k / a \tau^{1 / 2}$ [i.e., putting $(1+a \tau)^{1 / 2} \approx(a \tau)^{1 / 2}$ in Eq. (A2)]. After the $\xi$ integration, the equivalent of Eq. (A5) is then

$$
\frac{1}{2 D} \int_{0}^{2 \pi}|\cdots|^{2} d \theta_{k}=\frac{16 \pi^{3} a^{1 / 2} f^{2}(\rho)}{\Lambda^{2}(\rho) \tau^{3 / 2} k} \exp \left(\frac{-2 k^{2} \nu}{3 a}\right) \text {. }
$$

Repeating the steps giving Eq. (A7) and putting $\tau_{1} \rightarrow 0$, $\tau_{2} \rightarrow \infty$ we find

$$
\begin{aligned}
E_{s_{2}}(k)= & \frac{2 \pi N a^{1 / 2}}{k^{2}} \exp \left(\frac{-2 k^{2} \nu}{3 a}\right) \\
& \times \int_{0}^{\infty} \int_{\rho_{1}(\tau)}^{\infty} \tau^{-1 / 2} \frac{|f(\rho)|^{2} \rho}{|\Lambda(\rho)|} d \tau d \rho,
\end{aligned}
$$

where we have left the lower limit of integration $\rho_{1}$ an unspecified function of $\tau$ since putting $\rho_{1}=0$ gives a divergent $\tau$ integral. As the spiral vortex rolls up, the spacing between turns at time $t$ and radius $r$ can be shown to be

$$
\delta r \approx \frac{2 \pi}{|\Lambda(\rho)| \tau \sqrt{(1+a \tau)}} \approx \frac{2 \pi}{|\Lambda(\rho)| a^{1 / 2} \tau^{3 / 2}},
$$

where recall that $(\rho, \tau)$ are related to $(r, t)$ by Eq. (5). Thus if we track a material particle in the spiral, the distance between turns reduces owing to two distinct effects, first the differential rotation which tightens the spiral through a winding process, and second, through the compressive radial strain.

We now introduce a cutoff approximation by assuming that at fixed $k$ and $\tau$, the only portion of the spiral that can 
contribute to the spectrum is that for which $\delta r \geqslant 2 \pi / k$. At this $(k, \rho)$ the inner portion, for which $\delta r<2 \pi / k$, will contribute, by coarse graining, only to the axisymmetric spectrum $E_{0}(k)$. From Eq. (A11) this then fixes $\rho_{1}$ in Eq. (A10) as the solution of the equation

$$
k \sim\left|\Lambda\left(\rho_{1}\right)\right| a^{1 / 2} \tau^{3 / 2} .
$$

Exchanging the limits of integration in the $\tau, \rho$ plane in Eq. (A10) and using Eq. (A12) then gives

$$
\begin{aligned}
E_{s_{2}}(k)= & \frac{2 \pi N a^{1 / 2}}{k^{2}} \exp \left(\frac{-2 k^{2} \nu}{3 a}\right) \\
& \times \int_{0}^{\infty} \int_{0}^{\tau_{2}} \tau^{-1 / 2} \frac{|f(\rho)|^{2} \rho}{|\Lambda(\rho)|} d \rho d \tau,
\end{aligned}
$$

where $\tau_{2}=\left(k /|\Lambda(\rho)| a^{1 / 2}\right)^{2 / 3}$. Evaluating the $\tau$ integral then leads to

$E_{s_{2}}(k)=4 \pi N a^{1 / 3} k^{-5 / 3} \exp \left(-\frac{2 \nu k^{2}}{3 a}\right) \int_{0}^{\infty} \frac{|f(\rho)|^{2} \rho d \rho}{|\Lambda(\rho)|^{4 / 3}}$.

(A14)

Equation (A14) does not exactly agree with the first term of Eq. (29), differing by a constant factor $\Sigma_{1}^{\infty} n^{-4 / 3} / 3 \approx 1.27$.

\section{APPENDIX B: VORTICITY MOMENTS}

PS calculate the $\overline{\omega^{2 p}}$ moments by substitution of Eqs. (2)-(8) into Eqs. (15) and expansion of the $2 p$ th power of the Fourier series. Here we give a simpler intuitive derivation for $p \geqslant 2$. using Eq. (A1). We write

$$
\begin{aligned}
\overline{\omega^{2 p}} & =N \int_{A} \int_{\phi / a}^{\infty}(1+a \tau)^{2 p-1} \hat{\omega}^{2 p} d \tau d A, \quad p \geqslant 2, \\
& \approx N \int_{-\infty}^{\infty} \int_{S} \int_{\phi / a}^{\infty}(1+a \tau)^{2 p-1} \hat{\omega}^{2 p} d \tau d s d y,
\end{aligned}
$$

where $A$ refers to the vortex cross section and $S$ to integration along the shear layer. Using Eq. (A1) and performing the $y$ integration, and using $d s \approx \rho \Lambda(\rho) \tau d \rho$ gives

$$
\begin{aligned}
\overline{\omega^{2 p}}= & \frac{\sqrt{2} \pi(3 p)^{p-1 / 2} N}{\nu^{p-1 / 2} \sqrt{p}} \int_{\phi / a}^{\infty} \frac{(1+a \tau)^{2 p-1}}{\tau^{3 p-3 / 2}} d \tau \\
& \times \int_{0}^{\infty} \frac{|f(\rho)|^{2 p} \rho d \rho}{|\Lambda(\rho)|^{2 p-1}} .
\end{aligned}
$$

On performing the $\tau$ integral we find a result which agrees with Eqs. (82a) and (82b) of PS

$$
\begin{aligned}
\overline{\omega^{2 p}} & =\frac{N a^{3 p-5 / 2}}{\nu^{p-1 / 2}} \hat{L}_{2 p}(\phi) \int_{0}^{\infty} \frac{f^{2 p}(\rho) \rho d \rho}{|\Lambda(\rho)|^{2 p-1}}, \quad p \geqslant 2, \\
\hat{L}_{2 p}(\phi)= & \frac{2 \sqrt{2} \pi \Gamma[2 p](3 \pi)^{p-1 / 2}}{\sqrt{p}} \\
& \times \sum_{q=0}^{2 p-1} \frac{\phi^{q+5 / 2-3 p}}{(6 p-2 q-5) \Gamma[q+1] \Gamma[2 p-q]} .
\end{aligned}
$$

\section{APPENDIX C: SKEWNESS}

The following kinematic results are well known:

$$
\begin{aligned}
& \frac{35}{\overline{8}\left(\frac{\partial u}{\partial x}\right)^{3}}-\overline{c_{1} c_{2} c_{3}} \\
& \overline{\omega^{2}}=2 \int_{0}^{\infty} k^{2} E(k) d k \\
& \epsilon=15 \nu \overline{\left(\frac{\partial u}{\partial x}\right)^{2}}
\end{aligned}
$$

The following relations are consequences of the model. See Sec. V B of PS

$$
\begin{aligned}
& e_{1} e_{2} e_{3}=a^{3} / 4-a e_{r \theta}^{2}, \\
& e_{r \theta}^{2}=\omega^{2} / 4, \\
& \epsilon=\nu \overline{\omega^{2}}+3 \nu a^{2} .
\end{aligned}
$$

Combining Eqs. (C1), (C2), (C4), and (C5), we obtain

$$
\frac{35}{8} \overline{\left(\frac{\partial u}{\partial x}\right)^{3}}=\frac{a^{3}}{4}-\frac{a}{2} \int_{0}^{\infty} k^{2} E(k) d k \text {. }
$$

Dividing by $\left\{(\partial u / \partial x)^{2}\right\}^{3 / 2}$, we obtain using Eqs. (C2), (C3), and (C6),

$$
S_{3}=\frac{2}{35}(15)^{3 / 2}\left[4\left(\begin{array}{c}
\nu a^{2} \\
\epsilon
\end{array}\right)^{3 / 2}-\left(\frac{\nu a^{2}}{\epsilon}\right)^{1 / 2}\right]
$$

This is Eq. (62), used to calculate the skewness for Table III.

Alternatively, we can apply the Kármán-Howarth equation PS(40) to the model, to obtain another expression for the skewness

$$
\frac{35}{8}\left(\overline{\left.\frac{\partial u}{\partial x}\right)^{3}}=\frac{a^{3}}{4}-\frac{\nu}{2} \int_{0}^{\infty} k^{4} E(k) d k\right.
$$

Now if

$$
E(k)=C_{1} K e^{-K^{2} / 2}+C_{2} K^{-5 / 3} e^{-K^{2} / 6},
$$

with $C_{1}$ and $C_{2}$ independent of $K$, direct calculation [remembering that $\left.k=K / 2(a / \nu)^{1 / 2}\right]$ shows that

$$
\int_{0}^{\infty} k^{2} E(k) d k=\frac{\nu}{a} \int_{0}^{\infty} k^{4} E(k) d k,
$$

and (C7) and (C9) are the same.

Hence the two methods of calculating the skewness agree when the energy spectrum is of the form (C10). According to Eq. (37), this is the case for the $E_{0}$ contribution when $A$ is independent of $K$, which happens when $\tau_{2}=\infty$. 'The condition always holds, according to Eq. (40), for the asymmetric spiral contribution, which in general dominates. Note that Eq. (C11) depends sensitively on both the index of the power law and the coefficient and power in the exponential, and provides therefore a good check on the consistency of the model for $\Gamma(\infty)=0$ and $\tau_{2}=\infty$. 
When $\tau_{2}<\infty$, we find that Eq. (C11) is not satisfied. In this case, we note that PS(40) is not exact and its derivation from $\mathrm{PS}(39)$ neglects the left hand side of this equation which describes the time evolution of the vorticity. [There is a misprint in PS(39); the left-hand side should be multiplied by -1.$]$ Taking the time average of the left hand side of PS(39), which is equivalent to taking an ensemble average, we replace Eq. (C9) by

$$
\begin{aligned}
\frac{35}{8\left(\overline{\left(\frac{\partial u}{\partial x}\right)^{3}}=\right.} & \frac{a^{3}}{4}-\frac{\nu}{2} \int_{0}^{\infty} k^{4}\left(E_{0}(k)+E_{s}(k)\right) d k \\
& +\frac{1}{4} \frac{\partial}{\partial t_{1}} \int_{0}^{\infty} k^{2} E_{0}(k) d k \\
& -\frac{1}{4} \frac{\partial}{\partial t_{2}} \int_{0}^{\infty} k^{2} E_{0}(k) d k
\end{aligned}
$$

where $E_{0}(k)$ is defined as a function of $\tau_{1}$ and $\tau_{2}$ by Eq. (11). It is assumed that only the core contributes to the unsteady term because the spiral dies relatively quickly. The formula (C12) was employed to check the values of $S_{3}$ given in Table III. For the calculations reported presently agreement between Eqs. (C8) and (C12) to 8-10 decimal places was found.
'T. S. Lundgren, "Strained spiral vortex model for turbulent fine structurc," Phys. Fluids 25, 2193 (1982).

${ }^{2}$ D. I. Pullin and P. G. Saffman, "On the Lundgren-Townsend model of turbulent fine scales, ${ }^{\prime 2}$ Phys. Fluids A 5, 126 (1993).

3J. Jiménez, A. Wray, P. G. Saffman, and R. S. Rogallo, "The Structure of intense vorticity in homogeneous isotropic turbulence," J. Fluid Mech. 255, 65 (1993).

${ }^{4}$ T. S. Lundgren, "A small scale turbulence model," Phys. Fluids A 5, 1472 (1993).

${ }^{5} \mathrm{~S}$. Kida (private communication).

${ }^{6} \mathrm{~A}$. Vincent and M. Meneguzzi, "The spatial structure and statistical properfies of homngeneous turbulence," J. Fluid Mech. 225, 1 (1991).

${ }^{7}$ A. Abramowitz and I. S. Stegun, Handbook of Mathematical Functions (Dover, New York, 1965).

${ }^{8}$ G. F. Carrier, M. Krook, and C. E. Pearson, Functions of a Complex Variable (Hod, Ithaca, 1983).

"A. A. Townsend, "On the fine-scale structure of turbulence," Proc. R. Soc. Lundon Ser. A 208, 534 (1951).

${ }^{10} \mathrm{~A}$. Tsinober, E. Kitt, and T. Dracos, "Experimental investigation of the field of velocity gradients in turbulent flows," J. Fluid Mech. 242, 169 (1992).

"J. Buntine and D. I. Pullin, "Merger and cancellation of strained vortices," J. Fluid Mech. 205, 263 (1989).

${ }^{12}$ D. I. Pullin and J. D. Buntine, "Interactive dynamics of strained vortices," Proceedings of the 10th Australian Fluid Dynamics Conference, 10-15 December 1989 (University of Melbourne, Melbourne, 1989), Vol. 2, pp. 9.5-9.8.

${ }^{13}$ C. W. Van Atta and R. A. Antonia, "Reynolds number dependence of skewness and flatness factors of turbulent velucity derivatives," Phys. Fluids 23,252 (1980).

${ }^{14} \mathrm{~K}$. R. Steenivassan, "On the scaling of the turbulence energy dissipation rate," Phys. Fluids 5, 1048 (1984).

${ }^{15}$ Zhen-Su She and E. Jackson, "On the universal form of energy spectra in fully developed turbulence," Phys. Fluids A 5, 1526 (1993).

${ }^{16} \mathrm{~V}$. Yakhot and V. Zakharov, "Hidden conservation laws in hydrodynamics; energy and dissipation rate fluctuation spectra in strong turbulence," Physica D 64, 379 (1993). 